\title{
The Dynamic Interactive Pattern of Assimilation and Contrast: Accounting for Standard Extremity in Comparative Evaluations.
}

\author{
Paul Barker ${ }^{1}$, \& Roland Imhoff ${ }^{1,2}$ \\ 1Social Cognition Center Cologne, University of Cologne, Germany \\ 2Social and Legal Psychology, Johannes Gutenberg University Mainz, Germany
}

Accepted for publication at

Journal of Experimental Social Psychology

\begin{abstract}
Author Note
The corresponding author is Roland Imhoff (roland.imhoff@uni-mainz.de), Department of Social and Legal Psychology, Johannes Gutenberg University Mainz, Binger Str. 14-16, Mainz, 55122, Germany.

This project was supported by a DFG Grant (IM147/3-1) awarded to Roland Imhoff as part of the DFG Research Unit "Relativity in Social Cognition" (FOR 2150). The authors have no competing interests to declare.

Additional materials and data related to this manuscript can be found on the Open Science Framework page at: https://osf.io/sxq2c
\end{abstract}

Word count: 22071 


\begin{abstract}
Social judgments are often influenced by comparison to some standard in the environment, either moving the judgment closer (assimilating) to or away (contrasting) from this standard. Which direction this effect will take depends heavily on the relative standing of these standards on the judgment dimension compared to the target of the judgment. In previous research, items and comparison standards are often selected arbitrarily, ignoring or simplifying their influence substantially. The current work takes a fine-grained holistic curve fitting approach to measure response patterns across a wide range of standard extremities, showing that a narrower approach can pose limits to the generalizability and validity of inferences. A series of 8 experiments $(N=$ 4304) uncovered a dynamic interactive pattern between assimilative and contrastive forces on average, which proved sensitive to item level variation (1a, b \& c), but showed stability at the level of the facial judgment dimensions ( $2 \mathrm{a} \& \mathrm{~b})$. Finally, it revealed how the current approach can offer new insights into the role of other moderating variables, such as focusing on similarities versus differences ( $3 a \mathrm{a}$ b) and cross-category standards (4), which traditional approaches may miss. Implications for study design and theory are discussed.

Keywords: Social Comparison, Assimilation, Contrast, Facial Judgments, Construct validity.
\end{abstract}


To make more accurate judgments, people will often search the environment for a standard to which they can compare the target of this judgment. The influence these comparisons have on the judgments outcome is highly sensitive to contextual factors, such as the relative standing of the standard used relative to the judgment target. The likelihood of the judgment moving closer (assimilating) to or moving away (contrasting) from the standard is directly linked to their perceived extremity. More moderate standards are associated with a higher propensities towards assimilative effects and more extreme standards leading to contrast more often (Herr, 1986). However, what exactly constitutes a moderate or an extreme standard is not defined in the literature and represents a relatively arbitrary dichotomization of a truly continuous variable. Despite this tendency to oversimplify the continuous nature of a standard's standing in relation to the target, its influence will likely not be characterized by a sudden flip from one tendency to the other, but rather produce a gradual change as standards become more extreme. The current work will be the first to use a fine-grained manipulation in order to define this dynamic interactive pattern of assimilation and contrast across a wide range of standard extremity values, highlighting the fundamental connection between outcomes of comparative judgments the relative standing of their standards. In doing so, it will uncover strong judgment dependent variations in the comparison process and provide the first boundary conditions for the concept of 'extreme' for a number of facial dimension (Extraversion, Trustworthiness, and Dominance). Furthermore, it will show how influential moderating variables, such as comparative foci and shared category membership, affect these judgment patterns in a unique way, which could be missed by a more narrow approach leading to incorrect inferences.

Whenever we encounter someone new, we make instant judgments about them on a large number of dimension based on very little information. For instance, stereotypical notions can 
create deeply ingrained ideas of what others should look like (Imhoff et al., 2013), with judgments made on a variety of traits based solely their facial features (Todorov et al., 2015) within the first 100ms of an encounter (Willis \& Todorov, 2006; Ballew \& Todorov, 2007). Social judgments like these are often not made in absolute terms, but relative to some standard present in the environment (Festinger, 1954). Whether someone is athletic or intelligent can only be considered in relation to how well others fare in these domains. In this way, most social judgments are to some degree comparative in nature (Kahneman \& Miller, 1986). Which standard is used as a comparison is far from inconsequential, leading to measurable shifts in the final judgment (Mussweiler, 2003). Recent research has shown that this is no different for facial judgments (Barker et al., 2020) emphasizing the fundamentally comparative nature of human judgment (Dunning \& Hayes, 1996).

In broad terms, the integration of comparison information can logically only affect the final judgment in one of two ways; either the judgment is moved closer towards the standard (i.e., an assimilation effect) or it is pushed away from the standard (i.e., a contrast effect; e.g. Mussweiler, \& Strack, 1999; Schwarz, \& Bless, 1992a). Predicting which of these effects is more likely to occur for a given judgment is a relatively complex task and has led to the formation of a number of rivalling models, each attempting to explain the process in a distinct manner. One prominent model of comparative outcomes is the Selective Accessibility Model (SAM; Mussweiler, 2003), which states that the most fundamental determinant of the comparative outcome is a dichotomous initial holistic assessment of whether the target and the standard are similar or dissimilar. Depending on this assessment, a process of biased hypothesis testing will follow, where information congruent with this assessment will become more accessible and influential in the judgment formation. For instance, if the target and standard are 
seen as similar, information about the target that is similar to the standard will become accessible, leading to assimilation. If, on the other hand, one sees the two as dissimilar, knowledge consistent with this hypothesis of dissimilarities will be more influential leading to contrast effects.

In this way, any variable that affects this initial assessment will also affect the final outcome. Directly manipulating this assessment by asking or priming respondents to focus on either similarities or dissimilarities has, indeed, been found to influence anything from self(Mussweiler, 2001) and social judgments (Corcoran et al., 2009), to affective reactions (Epstude \& Mussweiler, 2009), and evaluative pairings (Corneille et al., 2009). This influence is not only an integral part of the SAM, but has also been acknowledged as a particularly powerful moderator by rivalling theories of comparative judgments (e.g., the Inclusion/Exclusion model; Schwarz \& Bless 2007). Comparative foci, therefore, seem to be an important aspect of the way in which humans make comparative judgments, with the SAM proposing that the most natural state is one in which similarities are sought and assimilation is the result (Mussweiler, 2003; Mussweiler \& Epstude, 2009). This is thought to be the case due to the fact that any comparative judgment must logically start with a search for similar structurally alignable features before these features can be compared (Markman \& Gentner, 1993; Gentner \& Markman, 1994). In line with this idea, empirical work has shown the distinct processing advantage of focusing on similarities which might explain why this bias exists (Corcoran et al., 2011).

Other contextual variables can, however, move this natural tendency for assimilation effect towards a state where dissimilarity judgments and contrast become more dominant. One such variable is the perceived extremity of the standard with regard to the judgment dimension being considered. The unique aspect of this moderator is that it relates directly to the informational 
content of the comparison standard that one is using. Therefore, this moderator is necessarily present and considered if any comparative effect is to occur at all. Within the framework of the SAM, the effect of this essential moderator is again down to the way in which it affects the initial assessment. The more extreme a standard is, the more likely it is to be seen as dissimilar, which in turn leads to contrast effects. On the other hand, more moderate standards will likely result in judgments of similarity and assimilative effects (e.g., Herr, 1986; Herr et al., 1983; Mussweiler et al., 2004a). Although other models propose slightly different mechanisms (e.g., representativeness and the feature overlap; IEM, Schwarz \& Bless, 1992b), including the internal aggregation of these dual forces prior to the final response, they do not contest the expected pattern of assimilation to moderate and contrast from extreme standards in general.

\section{What constitutes an extreme standard?}

Despite the broad acknowledgment of its moderating role in the comparison process, no attempts have been made to concretely define the boundaries regarding when a standard is extreme enough to produce contrast. Instead, the lack of guidelines has led researchers to use substantially varying ways of operationalizing this concept. Some investigations use well-known celebrities and imaginary characters, often resulting in only the most radical exemplars in history qualifying as extreme (e.g., Adolf Hitler or Santa Claus; Herr 1986), thus, providing little information about when contrastive effects are thought to first arise. Others have used hand crafted vignettes reflecting distinct levels of the judgment trait (Mussweiler et al., 2004a), but with little objectivity regarding the relative distance of the standards they are meant to manipulate. More recent attempts have attempted to use more objective selection criteria based on the number of standard deviations the standards were removed from the target (Barker et al. 2020). However, the absence of conceptual boundaries resulted in the rather arbitrarily chosen 
distances of $1 S D$ for moderate and $4 S D$ for extreme standards. Furthermore, these standards did not result in consistent evidence for the theoretically presumed pattern across different judgments. Instead large inter-item variation ranging from the expected pattern (for judgments related to extraversion), to exclusively assimilation effects (trustworthiness), only contrast effects (competence and dominance), or even simply null effects (likability) were uncovered.

In light of the variability between these studies, one may wonder if the discrepancy in outcomes could simply be ascribed to the insufficient calibration of the standard's extremity to produce the expected pattern in the latter work. In addition, the precise point at which assimilation turns to contrast, and with it what may be conceptualized as "extreme", may also depend on the judgment that is being considered. For instance, consider the unexpected pattern found for judgments of dominant behavior in Barker et al. (2020) where contrast occurred for extreme standards of $4 S D$, but also for standards as moderate as $1 S D$ from the target. It may well be that for this judgment specifically, rather than the moderation of extremity being absent altogether, the seemingly moderate standard was simply already too extreme to produce assimilation. A similar argument can be made if an extreme standard were found to produce assimilation effects, as it may have just not been extreme enough. With this in mind, it is not surprising that researchers sometimes instead opt to use the most extreme exemplars (e.g., Herr, 1986). However, some evidence suggests that even these may produce assimilation effects under circumstances in which they are construed as representing the trait dimensions they epitomize (Philippot, et al., 1991) or if they are seen as too extreme to be relevant for comparison (Lockwood \& Kunda, 1997).

These issues can be traced back to a simplified conceptualization of the relative standing of the standard as dichotomous rather than the continuous variable it is. This creates the illusion of 
a clear distinction between 'moderateness' and 'extremeness' that can be used to separate these categories precisely. The reality, on the other hand, is that the relative standing is continuous in nature. The dichotomous terms, therefore, necessarily refer to a wide range of values, which should be specified and sampled from broadly. Failure to do so leaves post-hoc judgments of the appropriateness of the operationalization within the current judgment context as an ever present alternative explanation for unexpected findings, limiting investigations to mere tests of auxiliary theories of operationalization (Meehl, 1990). To address these shortcomings and offer stronger tests of theory, the extremity variable will first need to be redefined in a continuous manner and with it we will need to reconsider the way in which the two ostensibly conflicting effects of assimilation and contrast interact at each point in this new range of values.

\section{The dynamic interactive pattern of assimilation and contrast}

Some accounts propose that dual forces can be at play simultaneously during the formation of a single judgment (e.g., assimilation for lateral comparisons in the IEM; Schwarz \& Bless 2007), naturally implying an internal aggregation of the opposing tendencies of assimilation and contrast. Other theoretical accounts, such as the SAM (Mussweiler 2003), view the comparative process as dichotomous in its outcome on the level of an individual judgment (i.e. either assimilation occurs or contrast does, but not a combination of both in a single judgment). However, even in these cases, natural fluctuations in the initial state of the comparison process will result in the both assimilative and contrastive judgments being present on aggregate across many similar judgments. Hence, moderating variables, such as the extremity of the standard, should only be seen as increasing the likelihood of one of the two outcomes to occur for a given judgment, rather than precluding individual judgments from still following the alternative route. This is generally the case regardless of the theoretical importance of a given moderator as most 
psychological variables cannot be directly manipulated, but are instead operationalized imperfectly using some instrumental manipulation. These instrumental variables, though intended to manipulate the latent moderating variable as effectively as possible, are themselves merely tangentially connected to the theoretical moderator and do not have perfect construct validity (Cronbach \& Meehl, 1955; Campbell, 1957; Cook \& Campbell, 1979). Therefore, these instrumental variables will affect the theoretical moderator imperfectly and only for a finite number of cases, as they remain subject to both random and unintended systematic influences.

In addition, the opposing nature of the possible outcomes that are expected, assimilation and contrast, will on aggregate suppress one another to varying degrees as the likelihood of either increases or decreases with the changing context. In Figure 1, the results of a simulation based on the more restrictive model (assimilation or contrast, but no combination) are presented to demonstrate what this logic entails in a simplified scenario where the strength of contrast and assimilation are equal and linear, but the propensity for the former increases as standards move from more moderate to extreme ${ }^{1}$. In the top-left panel we see single judgments, each represented by a single dot, which follow either an assimilative (dark grey triangles) or contrastive (light grey crosses) pattern. The top-right panel shows how the resulting averages (bars) obscure any separation between these two opposing patterns and instead reflect the aggregated interplay of the outcomes on aggregate. The aggregated pattern broadly represents a lying S-shape and can be described relatively precisely by a cubic function with a positive linear (capturing the initial assimilative bump) and a negative cubic term (reflecting the increasingly contrastive tendencies

\footnotetext{
${ }^{1}$ This basic simulation assumes a linear increase in the strength of comparative effects of $B=0.3$ and a quadratic increase in propensities for contrast, with both terms subject to significant participant variation $\left(\sigma_{\beta}^{2}=.02, \sigma_{p}^{2}=1\right)$ and measurement error $\left(\sigma_{\varepsilon}{ }^{2}=1\right)$. However, the overall S-shaped pattern is not dependent on these exact parameters as long as both opposing effects are assumed to be linear functions of the relative distance and the propensities reflect the expected moderation. The R-script for this illustrative example can be found in the additional materials.
} 
later on), as is reflected in the bottom left panel with the expected curve in isolation on the bottom right.
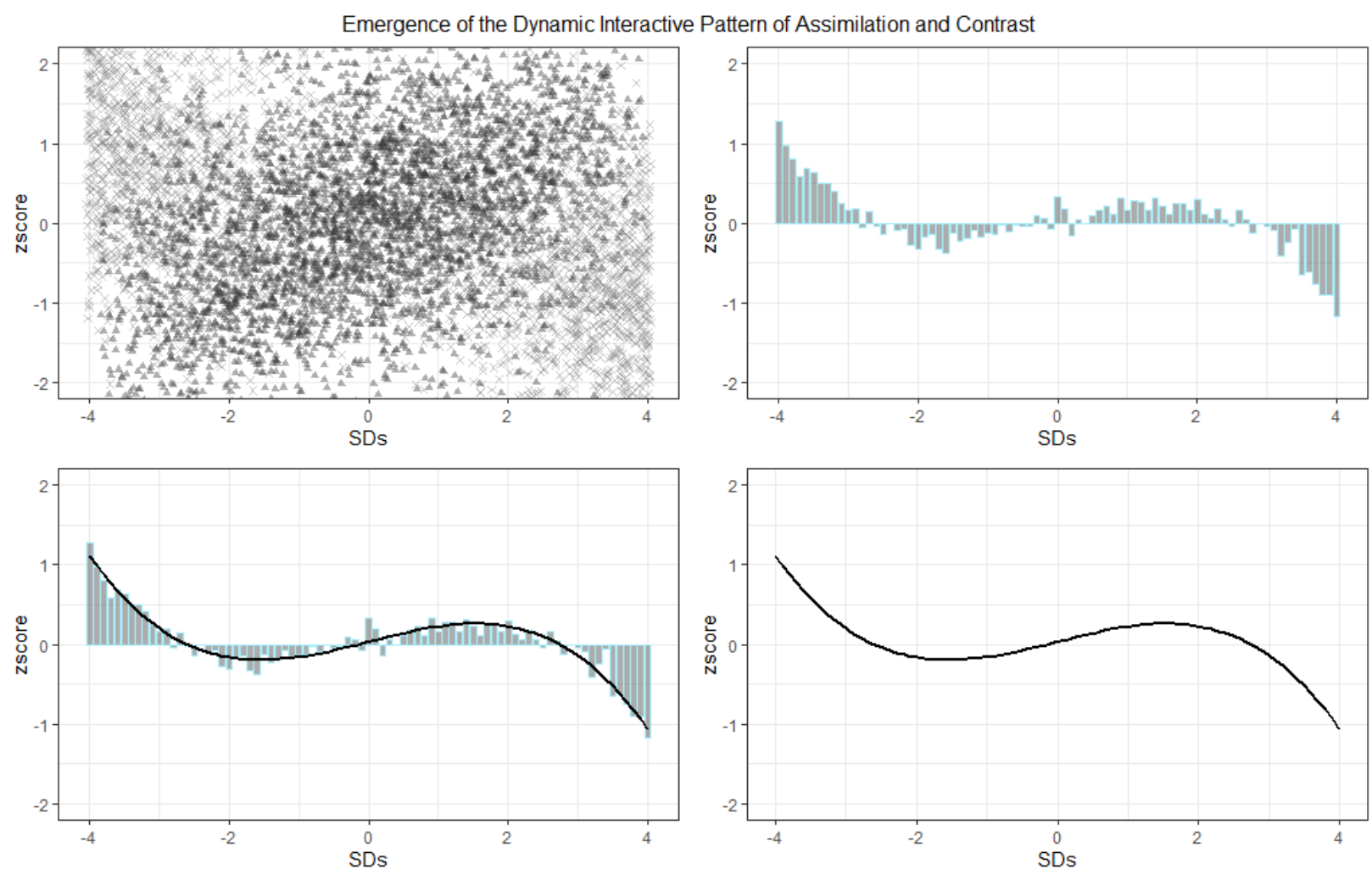

Figure 1. Assimilative (dark grey triangles) and contrastive (light grey crosses) individual responses resulting in a cubic S-shaped pattern as a function of increasing standard extremity when averaged. A z-score of 0 represents a judgment made with a completely neutral standard $(0 S D)$

Figure 2 shows a more exaggerated and illustrative depiction of the predicted dynamic interactive pattern of assimilation and contrast. Here we can better see how, on aggregate, the influence of assimilation is dominant for the most moderate standards and appears to increase in strength in both directions as the standards become more extreme. Once some peak is reached (indicated in the figure by the Point of Maximal Assimilation; PMA), the dominance of assimilative effects on average will start to wain as contrastive tendencies become ever more likely. The increasing prevalence of these contrast effects on a judgmental level will eventually start to rival the assimilative judgments until the aggregated strength of both outcomes 
effectively cancel each other out. This is indicated at the position where the S-shaped curve crosses the $\mathrm{x}$-axis in Figure 2. Every standard before this point, might be thought of as residing within the Window of Assimilation (WoA), a band of standard extremities where assimilation effects are on average dominant. The blue area under the curve within the WoA, which we will refer to as the Area of Assimilation (AoA), represents the cumulative strength of the aggregated outcome across the range of values in which assimilation is the dominant force. Moving past the turning point where both effects are in perfect equilibrium, assimilation will now become increasingly rare whereas contrast effects will become dominant, with consistent aggregated contrast effects presenting themselves for these more extreme standards.

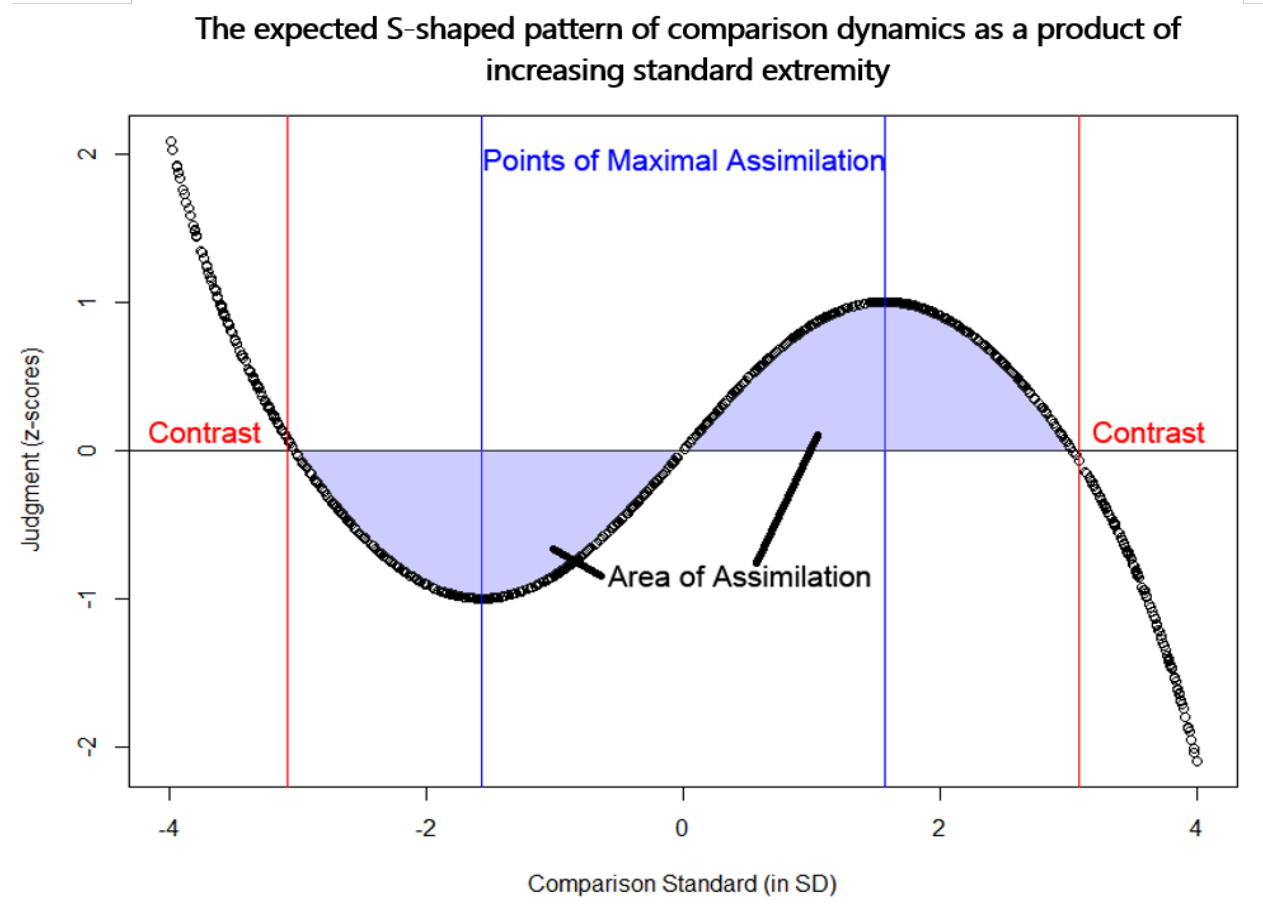

Figure 2. Some key characteristics of the S-shaped Dynamic interactive pattern. The $\mathrm{x}$-axis again represents the judgment that would be made with a completely neutral standard $(O S D)$

With this dynamic pattern in mind, a new issue emerges for the interpretation of the existing literature. Not only can any standard be deemed too extreme (or not extreme enough) in a post-hoc fashion when it has resulted in a contrastive (an assimilative) pattern, such critiques 
can likewise be presented to legitimately explain null effects. Without measuring across multiple standard extremities, the absence of assimilative and contrastive effects on average could reflect a state in which the two tendencies are in perfect equilibrium. These phantom comparisons can be found if the standards used are near points, where the graph crosses the axis. This means that any findings that do not account for the continuous nature of standard extremities will either confirm the predicted data pattern or may be deemed as theoretically inconsequential, since any failure to confirm the predictions is easily attributed to the insufficient calibration of the extremity variable. Therefore, the selection of single points or narrow windows of standard extremities can never suffice for strong tests of theory. Moreover, the lack of broader standard selection is not only prohibitive to the advancement or falsification of theories of comparison, but with a reversed logic it also strictly limits the generalizability of existing findings to the exact operationalization of the standard used in the investigation. This makes it exceedingly difficult to meaningfully compare results across studies and, as a result, also means that even attempts to summarize the existing literature using meta-analytical techniques may offer results that are theoretically meaningless unless they manage to account for the exact standings of the standards used within all the involved studies.

\section{The context dependency of comparisons}

In light of this complex dynamic that underlies the influence of standard extremity, it becomes evident that no investigation can make precise predictions if this variable is not accounted for. Moreover, the exact shape of the interactive pattern across the spectrum of extremity values itself is not static, but inextricably linked to the context in which the judgments are made. Any variable that alters the propensity for one outcome over the other will also affect the equilibrium between both comparative tendencies at each point in this spectrum. Another 
basic simulation can illustrate what this means for the dynamic interactive pattern as a whole when a hypothetical dichotomous moderator is introduced ${ }^{2}$. Looking at the results presented in Figure 3, the top-left panel shows how the individual responses still appear largely the same with no uniform shift from contrast to assimilation between the control condition (light grey crosses) and the experimental condition (dark grey triangles). However, when we look at the averages and the comparative pattern as a whole, using a cubic function, a clear shift in the turning points as well as the associated WoA and AoA become clear.
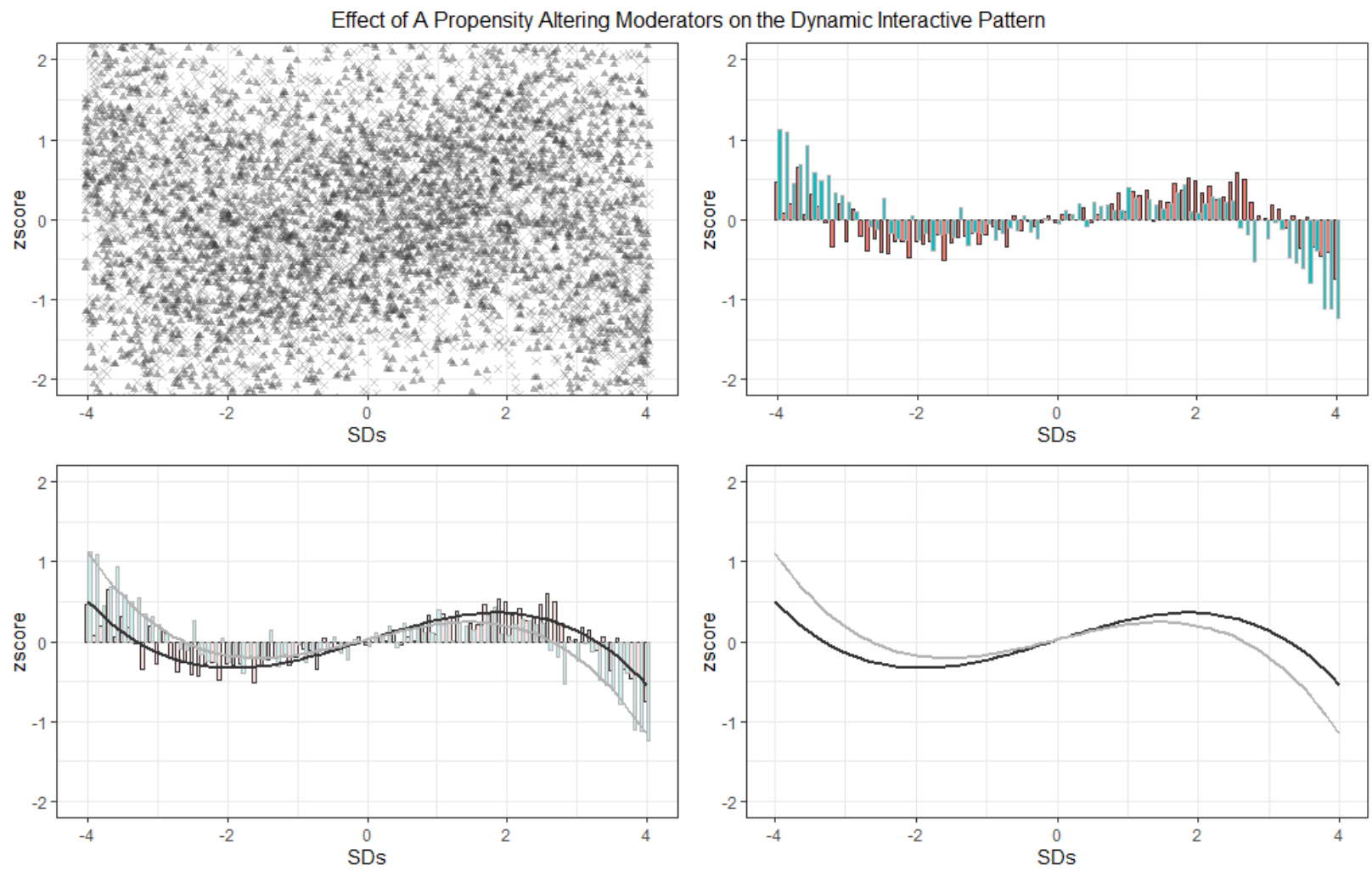

Figure 3. Simulated responses, averages, and the fitted cubic curves under a control condition (light grey crosses) and when the proportion of assimilation is increased by $30 \%$ (dark grey triangles). A z-score of 0 represents a judgment made with a completely neutral standard $(0 S D)$

\footnotetext{
${ }^{2}$ The same parameters were used as in the previous illustrative example, but with the addition of a between subject condition that uniformly increased the propensity for assimilation over contrast at each point by $30 \%$. The script can again be found in the additional materials.
} 
Hence any contextual variable that influences the propensity of one outcome to occur more frequently than the other will produce a similar shift in the comparative pattern. This may include the judgment itself (Barker, et al., 2020) if individual items have varying likelihoods of eliciting either outcome. Moreover, even theoretically strong moderators, like the initial holistic assessment that theoretically should lead to assimilation or contrast, will in practice lead to a similar shift rather than a sudden flip in outcomes due to the imperfect construct validity of its manipulations (Cook \& Campbell, 1979). Instead, a focus on differences rather than similarities would in practice be expected to shallow the curve for more moderate standards, decrease the AoA, and narrow the WoA. Note that this need not entail an absence of assimilation at every point on the scale, nor would inducing a similarity focus necessarily lead to assimilation at every point. In fact, an important characteristic of the interactive pattern is that the cumulative comparative effect is zero (i.e. assimilation and contrast cancel each other out) at the boundaries of the WoA (i.e. the points where the curve dissects the x-axis). Comparative judgments made in reaction to standards at this point would likely lead to apparent null effects, more aptly described as phantom comparisons. As shown in Figure 3, the standard extremities that produce these phantom comparisons will not necessarily be the same in all contexts and experimental conditions, as the point of equilibrium shifts along with the comparative pattern as a whole.

This is not the only way in which a moderator can affect the dynamic interactive pattern. Instead its influence will depend on the unique way in which it influences both comparative effects respectively. For instance, some variables might increase the strength of both comparison outcomes, as has been suggested when more comparative mind-set overall is induced (Mussweiler \& Epstude, 2009), or decrease them until they are no longer measurable, as is thought to be the case in the presence of less informative standards (Gilbert et al., 1995). These 
influences, would not be expected to influence the WoA at all, but instead only increase or decrease the AoA by strengthening or weakening both comparative outcomes equally. Figure 4 illustrates this by showing the resulting change in the dynamic pattern when comparative effects are halved in strength due to a different hypothetical moderator that produces weaker comparisons for both tendencies.
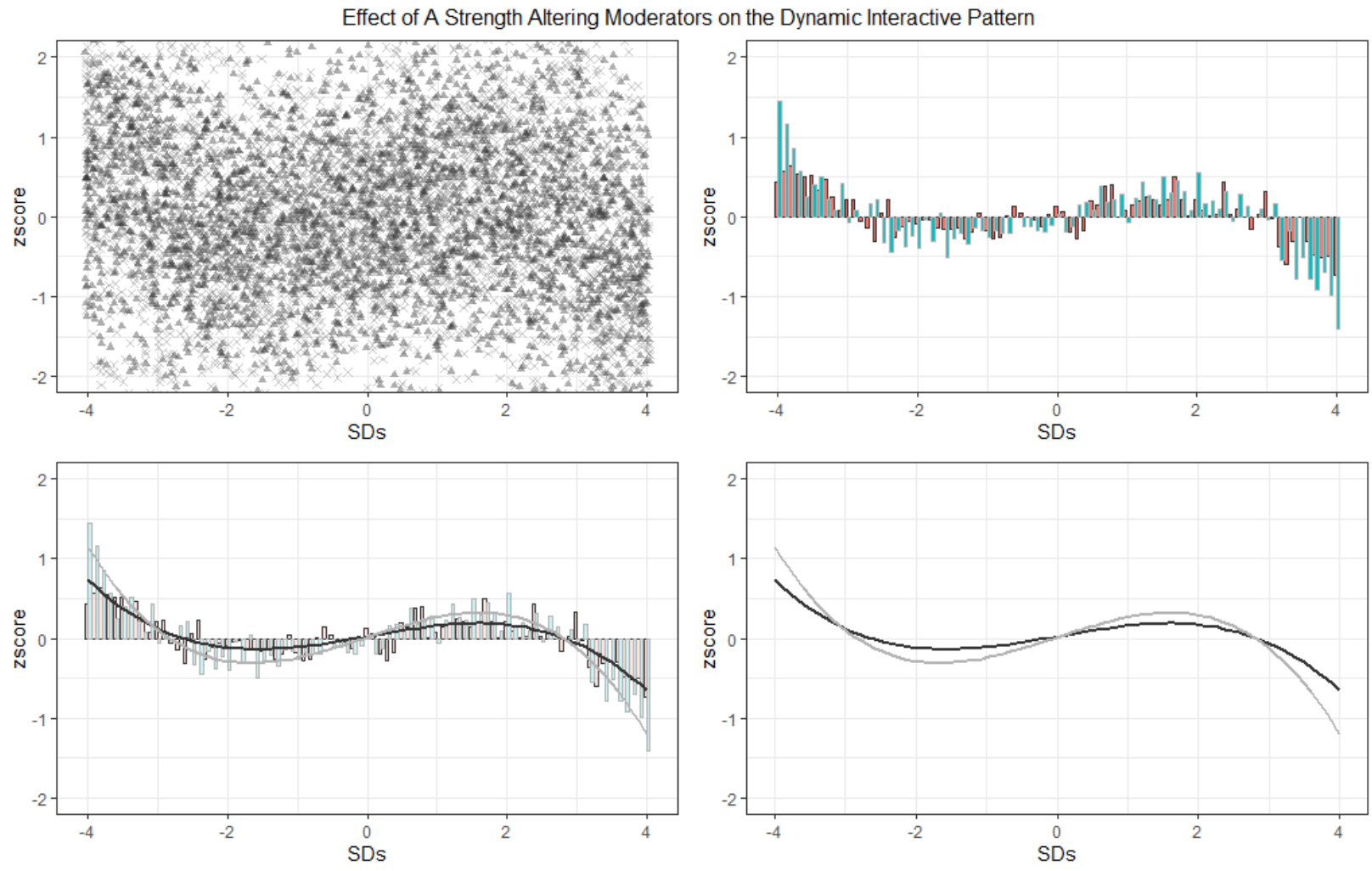

Figure 4. Simulated responses, averages, and the fitted cubic curves under a control condition (light grey triangles) and when both tendencies are attenuated by $30 \%$ (dark grey crosses). A z-score of 0 represents a judgment made with a completely neutral standard $(0 S D)$

A closer look at these two patterns side by side reveals that only considering a single measurement at the some point in the spectrum can produce seemingly identical results in both scenarios. For instance, a situation in which little to no comparative information is integrated in the final judgment, is almost identical to one in which assimilative and contrastive tendencies are in perfect equilibrium. Taking a more narrow approach may, therefore, lead to misspecification 
of a variable's influence or erroneous conclusions regarding empirical support. This is even the case for large aggregated data-sets made up of multiple studies. For instance, a recent metaanalysis by Gerber et al. (2018) attempted to summarize the last 50+ years of comparative research, but only found consistent evidence of contrast overall, while little to no evidence was found for assimilation, depending on the publication bias estimator, even under a similarity focus. However, due to the previously noted lack of consideration of the extremity of standards across the literature, this fundamental moderator could not be included by the researchers. As a result, the reported findings are a snapshot of the dynamic pattern and merely reflective of the most prevalent comparison contexts in the studies from which the data were taken. Therefore, the conclusions might not reflect the true state of the comparison process across different standard extremities. Indeed, comparison standards regularly used in the literature may simply be sampled close to the turning point under a similarity focus, while they are firmly beyond this point for the dissimilarity focus. As we have seen in Figure 3 and 4, this scenario would be indistinguishable from one in which true null effects occur as long as the extremity of the standards used is not modelled appropriately.

\section{The present research}

To overcome the above-outlined issues surrounding the use of a dichotomized operationalization of standard extremity, the current project will be a first attempt at modelling the hypothesized dynamic pattern of assimilation and contrast across a wide range of extremity steps. By doing so, it will provide a stronger tests of the moderating role of standard extremity and uncover the proposed dynamic interactive pattern. It will then show how this new approach can help avoid erroneous conclusions and offer more precise inferences regarding other 
moderators and their unique influence on the comparative pattern, specifically for the effects of a similarity vs. dissimilarity focus as well as shared vs. different category membership.

Although past constraints have made it difficult for researchers to achieve the precision necessary, new statistical techniques and recourses allow us to finally address these questions. By using a multilevel curve-fitting procedure to model the comparison outcomes across a large range of precisely manipulated standards, the current research will be able to test the theoretically predicted pattern as well as providing a more exact definition of what constitutes an extreme standard to guide future research. The expected S-shape of this pattern can be described parsimoniously by a cubic function with a negative cubic term, indicating increasingly frequent contrast for more extreme standards, and a positive linear effect, indicating consistent aggregated assimilative effects for the more moderate standards. This approach lends itself to a critical test of the proposed effect as a failure to achieve this pattern within a reasonable range of comparison standards (e.g., from -4 to $+4 S D$ s) now speaks against the universality of the theory itself, not against the more or less arbitrarily chosen comparison standards. In addition, the descriptive pattern that accompanies the result will provide the most fine-grained picture of the dynamic interplay between assimilation and contrast to date, which will in turn help to form a first definition of the point at which assimilation becomes contrast. Later inclusion of additional moderators will show how these points, along with the related Window of Assimilation (WoA) and Area of Assimilation (AoA), shift and can act as metrics for novel tests of theory.

The current investigation will focus on the three facial dimensions that produced strongly varying comparison effects in previous work (Extraversion, Dominance, and Trustworthiness; Barker et al., 2020). In addition to allowing the further investigation of these unexpected variations and their causes, the domain of facial judgments simultaneously presents an excellent 
way of achieving the fine grained manipulation of standard extremity necessary to determine comparison patterns for multiple judgments in a standardized fashion. Indeed, faces allow for fast and unobtrusive presentation of comparative information. In addition, an almost endless supply of unique facial stimuli can now be easily created and precisely manipulated on a number of social dimensions with the use of data-driven algorithms (see Todorov et al., 2013) providing the numerous comparison targets and standards needed for the current endeavor. However, this does restrict the exact patterns presented here to the facial domain specifically, which should be kept in mind when interpreting the results presented throughout. Notwithstanding these issues, this domain offers a particularly suitable context to provide this first evidence of proposed dynamic interactive pattern and related shifts that occur across contexts.

An additional note here is that the paradigms employed in the social perception literature, which were used to initially calibrate the mapping of these facial dimensions, tend to measure the judgments one image at a time. In a departure from this convention, the current work will present two images alongside each other to evaluate the influence of the simultaneous comparative process in detail. Although this means that the results will be most relevant to the literature on comparative judgments specifically, they will also still have some relevance to the social perception literature since even judgments of isolated images are often made in comparison to some internal standard (Dunning \& Hayes, 1996) or a previous image (Kondo et al., 2013). These processes may similarly take on non-linear patterns as has already been reported for facial attractiveness ratings (Cogan et al., 2013). Even though such sequential effect will not be investigated here directly, the general framework described will offer a potential avenue for the investigation of this non-linearity in the social perception literature (for a similar approach to sequential judgments of numerical size see Barker \& Imhoff, 2021). 
The present research will investigate reactions to a wide array of comparison standards manipulated to differ on the dimensions of extraversion (Study 1a), trustworthiness (Study 1b) and dominance (Study 1c) with single judgment items used in Barker et al. (2020). To disentangle effects due to the specific items from effects of the judgment dimension proper, Study $2 \mathrm{a}$ and $2 \mathrm{~b}$ tested the hypothesized pattern for dominance (Study $2 \mathrm{a}$ ) and trustworthiness (Study 2b) with multiple items generated in a data-driven fashion. In addition, including items as random factors, these studies allow first clear guidelines for future research regarding the operationalization of a standards extremity in this context. Study $3 a$ and $3 b$ followed up on this to test the moderating role of the different comparative foci when explicitly induced. Finally, the paradigm's ability to clearly distinguish between simple equilibrium of comparative tendencies and their attenuation was critically tested Study 4, where comparison standards differed in whether they belonged to the same or a different racial category as the judgment target. Taken together these studies not only show the immediate need for broader item and standard selection in comparative research, but also provide at least one method which allows for this and can provide stronger tests of theory in the future.

All studies report full descriptions of power calculations, data exclusion, manipulations, and measures used. All sample sizes were determined before any data analysis. All studies were conducted in accordance with the 1964 Helsinki Declaration and its later amendments (WMA, 2001), as well as the 2016 American Psychological Association Ethical Principles of Psychologists and Code of Conduct (APA, 2017). As the studies did not involve deception, vulnerable populations, identifiable data, intensive data, or interventions they were exempt from ethical approval by the funding agency and host institution. Pilot studies, not directly described here, can be found in the additional materials and are referenced in the relevant sections. 
Furthermore, all anonymized $\mathrm{raw}^{3}$ and aggregated data, additional analysis details and supplemental materials for all studies can be found on the Open Science Framework page at: https://osf.io/sxq2c

\section{Studies 1a to 1c: Judgment Sensitivity of the Dynamic Pattern}

Using fixed positions for moderate $( \pm 1 \mathrm{SD})$ and extreme ( $\pm 4 \mathrm{SD})$ standards, previous work had observed only contrast for dominance, only assimilation for trustworthiness, and the predicted pattern as a function of extremity for extraversion judgments (Barker et al., 2020). This initial section will further investigate the judgment sensitivity of social comparative outcomes. Modelling the whole dynamic interactive pattern will reveal whether this variability is bound to the arbitrary selection of the standard extremities used in this previous work or if it represents a fundamental judgment sensitivity of the pattern as a whole. For this, three studies, closely related in their methodological approach, one for each of the facial dimensions will be presented. In the interest of brevity, a broad description of the methodological approach will be first offered here, before any minor variations and details are described for the individual studies in the separate sections.

Comparative Judgment Task (CJT). An expanded version of the Comparative Judgment Task (CJT; Barker et al., 2020) was used to investigate the hypothesized comparative patterns. Across 324 trials, participants judged a neutral target face on a particular judgment dimension using the number keys on their keyboards in an open-ended format. Open-ended items have been previously shown to elicit comparative processing without explicitly prompting participants to compare (Mussweiler et al., 2004b) and also avoid enforcing relative judgments

\footnotetext{
${ }^{3}$ Data about participants study area and gender are not included for lab studies nor are any timestamps for all studies to ensure the complete anonymity of participants. These data are available upon request with stricter sharing protections.
} 
themselves, which can be the case for many closed scales (i.e., each scale point of a Likert scale is subject to calibration in relation to the perceived range of judgment potential targets; Mussweiler \& Strack, 2000). At the same time, a second face filled the role of the comparison standard shown alongside the target. Unlike the target which was neutral with respect to the judgment dimension throughout the trials, these standards varied in their standing on the dimension, ranging from extremely high upward $(+4 S D$ from the mean of all faces in face space) to extremely low downward comparisons $(-4 S D)$. To ensure participants understand which face needed to be judged as the target, they had to identify the face labelled as the 'Judgment target' prior to making their judgments. In the current set-up, this was done by pressing either ' $\mathrm{Z}$ ' for the left (on the [masked] keyboards used this was the letter ' $\mathrm{Y}$ ' in the same location) or ' $\mathrm{C}$ ' for the right face, before the open-ended response was entered. This step also formed an attention check to exclude non-informative responses from the final dataset, see Appendix A for an example trial. In addition to the measurement instances used in previous CJT paradigms, the current set-up was significantly expanded to include 4 measurement instances for all $0.1 S D$ incremental extremity steps between $-4 S D$ and $4 S D$ away from the mean of all faces, resulting in a total of 81 steps and 324 trials.

Stimuli. To precisely investigate the judgment level variation described in Barker et al. (2020), the items that were pre-tested to relate to facial Extraversion, Dominance and Trustworthiness from this work were included; i.e., "How often does the target go out in 6 months?", "How many times does the target enforce his opinion in 6 months?", and "How many times does the target deceive somebody in 6 months?" respectively.

The facial stimuli needed for the CJT were created using a custom script built on the FaceGen SDK according to the process used in Todorov, et al. (2013). In this process, a unique 
random ID is first created after which it can be manipulated precisely across a range of facial dimensions to create numerous computer-generated facial images. The neutral IDs were then manipulated along the vector of facial extraversion, dominance, or trustworthiness to represent standards at every $0.1 S D$ step from neutral in a range from $-4 S D$ to $+4 S D$. This was done for four unique IDs at each of the 81 steps to produce a total of 324 unique facial pairs for each facial dimension, see Appendix B for examples. The neutral faces of each pair formed the judgment targets while the non-neutral faces acted as the various levels of comparison standards. The viewing distance was not controlled precisely, but was roughly $60 \mathrm{~cm}$. Stimuli were 220 pixel in height by 150 pixels, translating to a size of viewing angle of around $3.65^{\circ}$ horizontally and $5.36^{\circ}$ vertically on the 24 inch screen with a 1920 y1080 pixel resolution.

Procedure. In all studies, participants were recruited on campus at the University of Cologne, Germany, and were fully informed regarding the general procedure of the study and data storage policy before giving their consent and taking part in the study. In the first part of the study, some basic demographics, such as age, sex, and education were recorded, after which the CJT was explained in detail and participants conducted two practice trials. If participants had no remaining questions regarding the task they then started the main batch of 324 trials in random order. After completing the CJT trials, any additional materials were administered after which participants were debriefed and given their compensation.

\section{Study 1a: Extraversion}

The item related to extraversion was the first to be investigated using the CJT. Barker et al. (2020) found that this item was the only one that showed the expected assimilative and contrastive effect in response to the moderate (1SD) and extremity (4SD) standards that were used. The current study will attempt to model this pattern across the whole extremity spectrum. 
For all studies, the pattern we expect to see for these judgments if assimilation is more likely than contrast for moderate standards, and contrast is more likely than assimilation for more extreme ones, is an S-shaped cubic function as detailed in the introduction. More specifically the cubic function will be expected to have a positive linear effect, forming the initial aggregated assimilative bump, and a negative cubic term, showing the later increase in contrastive judgments and eventual dominance on average. If both assimilation and contrast are present on an aggregated level in the described pattern, we would also see a point at which the function crosses the intercept adjusted $\mathrm{x}$-axis. This is the point after which consistent contrast effects occur and, therefore, we can conceptually think of it as the lower boundary of what constitutes an extreme standard for this judgment. One must note that the absence of this aggregated assimilation or contrast in a cubic function, does not necessarily mean none took place on an individual judgment level, but merely that this tendency never reaches dominance over the other.

\section{Method}

Participants. To gain some indication of the sample size that would be necessary in the extended CJT paradigm, we assumed that the peak assimilative effect would be roughly the same size as the effect found for this judgment in Barker et al. (2020). Based on this, the linear effect was assumed to be around $B=0.17$ and the cubic effect around $B=-0.017$. Simulations with varying parameters showed that a sample size of roughly 80 participants would be needed in the repeated measures design with 324 trials to ensure at least $80 \%$ power to find the cubic function even when residuals and variation in the linear slope at the participant level was relatively large.

To leave some room for drop out, a convenience sample of 85 German speakers were recruited on campus at the University of Cologne to complete the 50 min study for a monetary 
reward of 10 euros. With the final sample being composed of $61.2 \%$ female participants and aged between 19 and 39 years $(M=25.41, S D=4.41)$.

CJT. As explained in more detail in the introductory section for Study 1a-c, participants will be presented with 324 pairs of facial images each. Participants will be asked to judge a neutral target face in a numerical open ended item relating to the extraversion dimension taken from Barker et al. (2020), which asks "How often does the target go out in 6 months?". The second face in the pair will not itself be judged but will be the standard ranging from $+/-4 S D$ away from the neutral target in $0.1 S D$ steps on the extraversion dimension, resulting in 81 steps repeated 4 times for 324 trial in total.

Data treatment. Non-numeric and empty responses made up $9.4 \%$ of trials while $8.8 \%$ showed a failed attention check leading to their exclusion in the analyses. The remaining scores were then used to calculate z-scores separately per participant to account for personal differences in response ranges. Z-scores above 3 or below -3 , or instances where no z-score could be calculated were removed, which was the case for $2 \%$ of trials. Due to co-occurrences of these criteria a total of $18.5 \%$ of the original trials were not used in the analyses. In the case of eight participants, this meant no usable trials remained, leaving the data of 77 participants to be used in the analyses.

\section{Results}

The main analysis was performed in R (Version 3.5.1; R Core Team, 2018) and consisted of a mixed models regression using a restricted maximum likelihood estimation (REML; using the lme4 package; Bates et al., 2015) with fixed effects for the extremity steps up to the third polynomials term, and with similar orthogonal uncorrelated random slopes and intercepts for participants to account for participant level variation in comparison patterns. To determine the 
confidence intervals and p-values for the fixed effects in this and all following studies,

Satterthwaite's approximations were used to estimate the appropriate degrees of freedom (using the lmerTest package; Kuznetsova et al., 2017; and the parameters package in R; Lüdecke et al., 2020).

Table 1

All fixed effects and related statistics from mixed model analysis

\begin{tabular}{|c|c|c|c|c|c|}
\hline & $B[95 \% \mathrm{CI}]$ & $S E$ & $d f$ & $t$ & $p$ \\
\hline \multicolumn{6}{|l|}{ Fixed effects: } \\
\hline $\mathrm{x}$ & $0.0191[0.0059 ; 0.0324]$ & 0.0068 & 127.321 & 2.826 & .005 \\
\hline$x^{2}$ & $-0.0012[-0.0037 ; 0.0012]$ & 0.0013 & 91.510 & -0.984 & .328 \\
\hline$x^{3}$ & $-0.0020[-0.0031 ;-0.0008]$ & 0.0006 & 290.346 & -3.350 & $<.001$ \\
\hline Intercept & $-0.0176[-0.0353 ; 0.0000]$ & 0.0090 & 162.513 & -1.957 & .052 \\
\hline
\end{tabular}

Results showed that in the cubic model, both the predicted positive first order and negative third order terms were present and significant (Table 1). The fitted values still described the expected S-shaped curve that would be expected with the presence of both assimilation and contrast (Figure 5).

Secondary analysis, using a slightly adjusted two lines test with the Robin Hood algorithm implemented to calculate the break point (Simonsohn, 2018) were run to also provide separate evidence of assimilation (i.e. an initial linear increase of z-scores as extremity increases) followed by increased prominence of contrast effects (i.e. a negative linear relationship after some peak has been reached). This procedure estimates a separate regression line for low and high values of the predictor, leaving more flexibility in the functional form that describes the relationship. However, to account for the clustered nature of the data a Mixed GAMM with random intercepts and linear slopes for participants was preferred (using the gamm4 package in R; Wood \& Scheipl, 2017) as were clustered robust standard errors by participant (using the sandwich package in R; Zeileis 2004; Berger et al., 2017). Robust LMM were initially also 
attempted to fit the full model, but were too large a strain on the memory capacity of the available hardware. The data set was also simplified for this analysis by disregarding the direction of the comparison with the steps now representing the absolute distance from the neutral target and Z-scores always reflecting assimilation when positive and contrast when negative. In line with the expectations, the results of this analysis showed both a significant average assimilative effect up to $0.8 S D, B=0.19, \mathrm{Z}=3.75, p<.001$, and a negative one beyond that, $B=-0.03, \mathrm{Z}=-3.89, p<.001$.

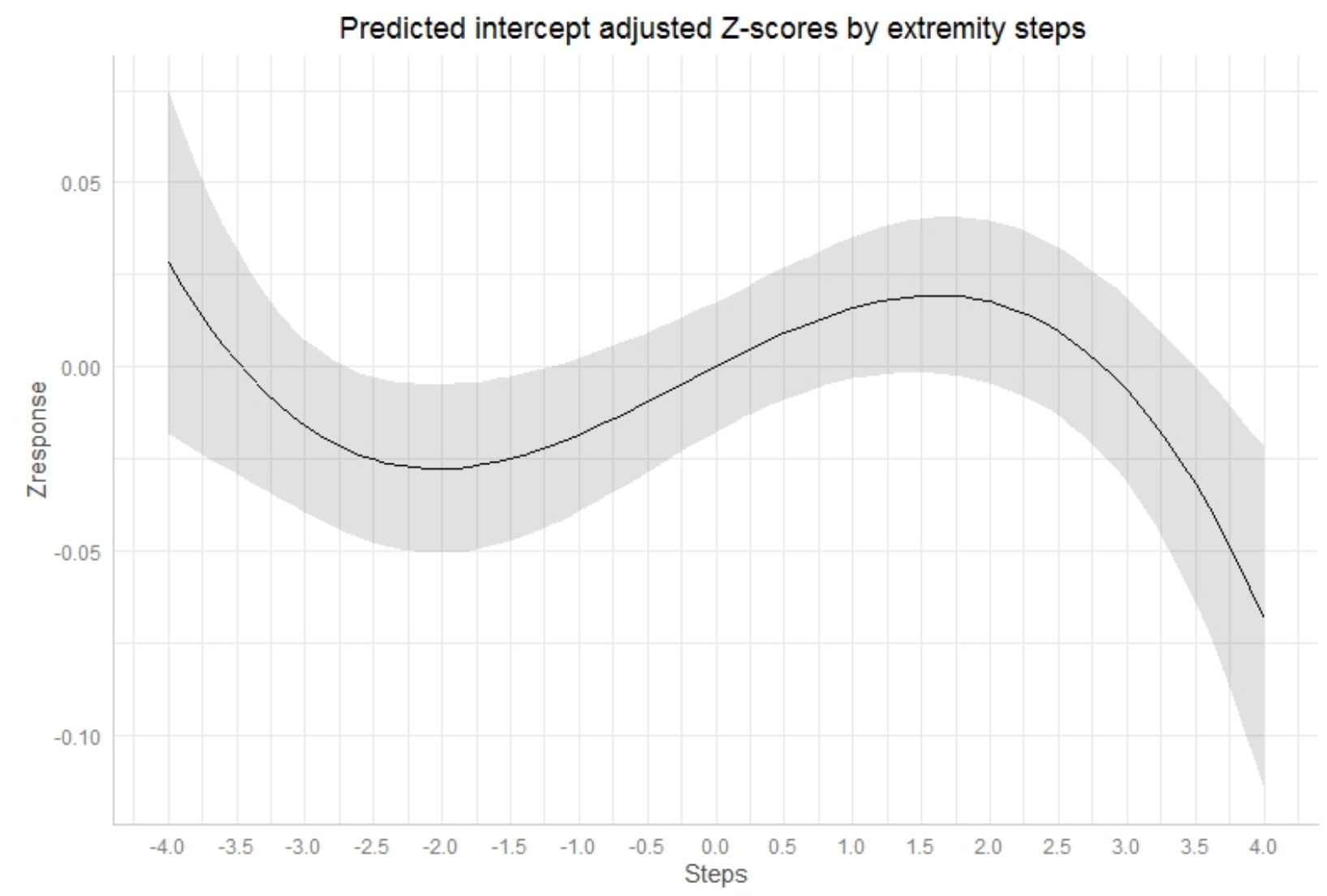

Figure 5. Predicted intercept adjusted marginal Z-scores for the extraversion dimension at each extremity step and predicted 95\%CI (created with the ggeffects package, Lüdecke, 2018)

The question remains whether the pattern is one of reduced assimilation only or contrast proper (i.e. is there a point at which judgments are on average consistently below the neutral judgment). For this, we first turn back to Figure 5, where one can see the curve clearly crosses 
the $\mathrm{x}$-axis (representing a judgment with a neutral standard) for both comparison directions, implying there is indeed a point at which the estimated marginal effect turns from consistent assimilation to contrast. To estimate these points and calculate their confidence intervals precisely, a simplified model using maximum likelihood estimation with only a random linear slopes for participants was used in a bootstrapping procedure with 3000 iterations to calculate percentile CIs (utilizing the boot package; Canty \& Ripley, 2019). For each iteration, the resulting polynomial curve was solved for zero where possible (with the polynom package; Venables et al., 2016). If an iteration only showed signs of assimilation across the range of measured instances the maximum measured step of $4 S D$ was returned, while if only contrast effects were present the returned value was set to the minimum value of $0 S D$. The returned values thus give a representation of the outer bounds of the window in which assimilation is the dominant tendency within the measured range. The procedure estimated these points to be as low as $-3.46 S D, S E=0.39$, Bias $=0.01,95 \% \mathrm{CI}[-4.00,-2.71]$, and as high as $2.82 S D, S E=0.41$, Bias $=-0.01,95 \% \mathrm{CI}[1.97,3.52]$, with the complete window of assimilation spanning $6.29 S D, S E=$ 0.50, Bias $=-0.02,95 \% \mathrm{CI}[5.38,7.01]$, indicating that there were clear signs of assimilation and contrast overall, although it did not provide evidence for a turning point for downward standards separately (as -4.00 was within the $95 \% \mathrm{CI}$ ). Nevertheless, the results taken together seem to convincingly support the expected pattern of assimilation to more moderate and contrast from more extreme standards for this judgment overall and the ability of the paradigm to detect both.

\section{Discussion}

This first implementation of the extended CJT successfully detected both assimilation and contrast effects in the expected S-shaped dynamic interactive pattern for a judgment that elicited both in previous work. Furthermore, the bootstrapping procedure successfully provided a first 
insight into the scope of the window of assimilation and what constitutes an extreme standard, although confidence intervals remained quite wide with the current sample size.

Related to this issue, one should also note the rather small size of the effects, which may partially be the result of the opposing effects that are at play and likely interfere with each other to some degree at any level of comparison since target extremity is but one of many moderators that affect the comparison outcome (Mussweiler, 2003). These issues could be exacerbated by the specifics of the current paradigm, which uses open ended responses and facial judgments of others that provide only minimal information for participants to base their judgments on. Additionally, comparisons are known to often be made in an egocentric manner with trait dimensions often understood idiosyncratically and based to some extent on one's own behaviors and characteristics (Dunning \& Hayes, 1996; Dunning et al., 1989). These issues could lead to larger variations in judgments overall and a lower reliability of the measure within the context of other-related facial judgments.

Nevertheless, the predicted pattern was still detected successfully, even with these caveats, highlighting the fundamental nature of comparative judgments, which can affect judgments even in the briefest of encounters. The next study will turn towards the judgment dimension of trustworthiness which did not produce any signs of aggregated contrast in prior work. With an increased sample size, the dynamic interactive pattern will be modelled once again to investigate if the potential judgment sensitivity of the comparative outcomes are limited to the standards extremities or show up across the extremity spectrum.

\section{Study 1b: Trustworthiness}

Unlike the judgment for extraversion, the judgment related to the facial dimension of trustworthiness was found to lead to only consistent assimilative tendencies (Barker et al., 2020). 
The fine-grained measurement of the Extended CJT can show if this specific judgment (i.e., "How many times does the target deceive somebody in 6 months?") increases the likelihood of assimilative effects resulting in the absence of aggregated contrast effects across the extremity spectrum and thereby confirming the judgment sensitivity of the dynamic interactive pattern.

Participants. Considering the small effect sizes and large CIs found for the turning point in Study 1a, the sample size for this study was roughly doubled for increased measurement accuracy. Therefore, 160 German speakers were again recruited on campus at the University of Cologne to complete the study for a monetary reward of 10 euros. One participant dropped out during the study, resulting in 159 completed cases being recorded. This final sample consisted of $62.9 \%$ females and was aged between 18 and 63 years $(M=24.09, S D=6.05)$.

CJT. The task remained identical to Study la with the exception of the open ended item, which now was the reverse coded item related to the trustworthiness dimension taken from Barker et al. (2020) that was worded as "How many times does the target deceive somebody in 6 months?".

Additional Measures. Age, sex, education and area of study were again measured as basic demographics in this study. In addition, the Iowa-Netherlands Comparison Orientation Scale (INCOM; Gibbons \& Buunk, 1999) was administered for exploratory reasons. The INCOM scale consists of 11 items $(\alpha=.77)$ that are averaged to create an INCOM score, with higher scores indicating an individual has a higher disposition to engage in social comparisons in daily life. Items in the scale focus on comparisons of one's own abilities or opinions with those of others. The scale was, therefore, included at the end of the study to explore the possibility that this construct might extend to broader tendencies for making comparison of others as well. As 
this measure did not produce any interesting findings in any of the studies the results will not be reported in this paper, but the relevant data are of course available in the supplemental materials.

Data treatment. As was the case in the previous study, trials with null responses or only non-numeric symbols were excluded from analyses (3.7 \% of trials), as well as trials in which the attention check was failed (7.1\%). Remaining scores were z-transformed per participant where possible after which the resulting z-scores were truncated above 3 and below $-3(2.6 \%)$. A total of $12.7 \%$ of all trials were excluded by these criteria. For 3 participants this meant none of their trials could be used, leaving 156 participants with data that was usable in the analyses. Due to the reversed nature of the judgment item, these scores were inversed so that higher scores reflected more trustworthy behavior to aid interpretation.

\section{Results}

Once again, a mixed models regression with REML estimation was implemented with polynomials up to the third degree for the extremity steps as fixed effects and the full orthogonal and uncorrelated random slopes and intercepts for each participants. The predicted negative third order term was again significant, as was the positive linear term consistent with the possibility of both assimilation and contrast (Table 2). However, it seems the estimated marginal z-scores indeed decreases with more extreme standards, but never cross the axis which would indicate consistent contrast never actually occurs within the measured range for this judgment (Figure 6).

Table 2

All fixed effects and related statistics from mixed model analysis.

\begin{tabular}{|c|c|c|c|c|c|}
\hline & $B[95 \% \mathrm{CI}]$ & se & $d f$ & $t$ & $P$ \\
\hline \multicolumn{6}{|l|}{ Fixed effects: } \\
\hline $\mathrm{x}$ & $0.035[0.0249 ; 0.0443]$ & 0.0050 & 210.423 & 6.966 & $<.001$ \\
\hline$x^{2}$ & $0.0012[-0.0006 ; 0.0029]$ & 0.0009 & 154.630 & 1.282 & .202 \\
\hline$x^{3}$ & $-0.0018[-0.0025 ;-0.0010]$ & 0.0004 & 1603.829 & -4.644 & $<.001$ \\
\hline Intercept & $0.0305[0.0179 ; 0.0430]$ & 0.0064 & 288.020 & -4.768 & $<.001$ \\
\hline
\end{tabular}


To see if separate evidence can be provided for the initial increase and apparent decrease in scores, the adjusted two lines procedure was implemented once again on a simplified unidirectional dataset. A significant initial linear increase signaling assimilation was found up to 1.9SD, $B=-0.03, Z=3.25, p=.001$. However, beyond this point the linear decrease failed to reach significance at the standard level, $B=-0.015, Z=-1.88, p=.06$, indicating the increased prevalence of contrast for more extreme standards remains unsupported for this judgment.

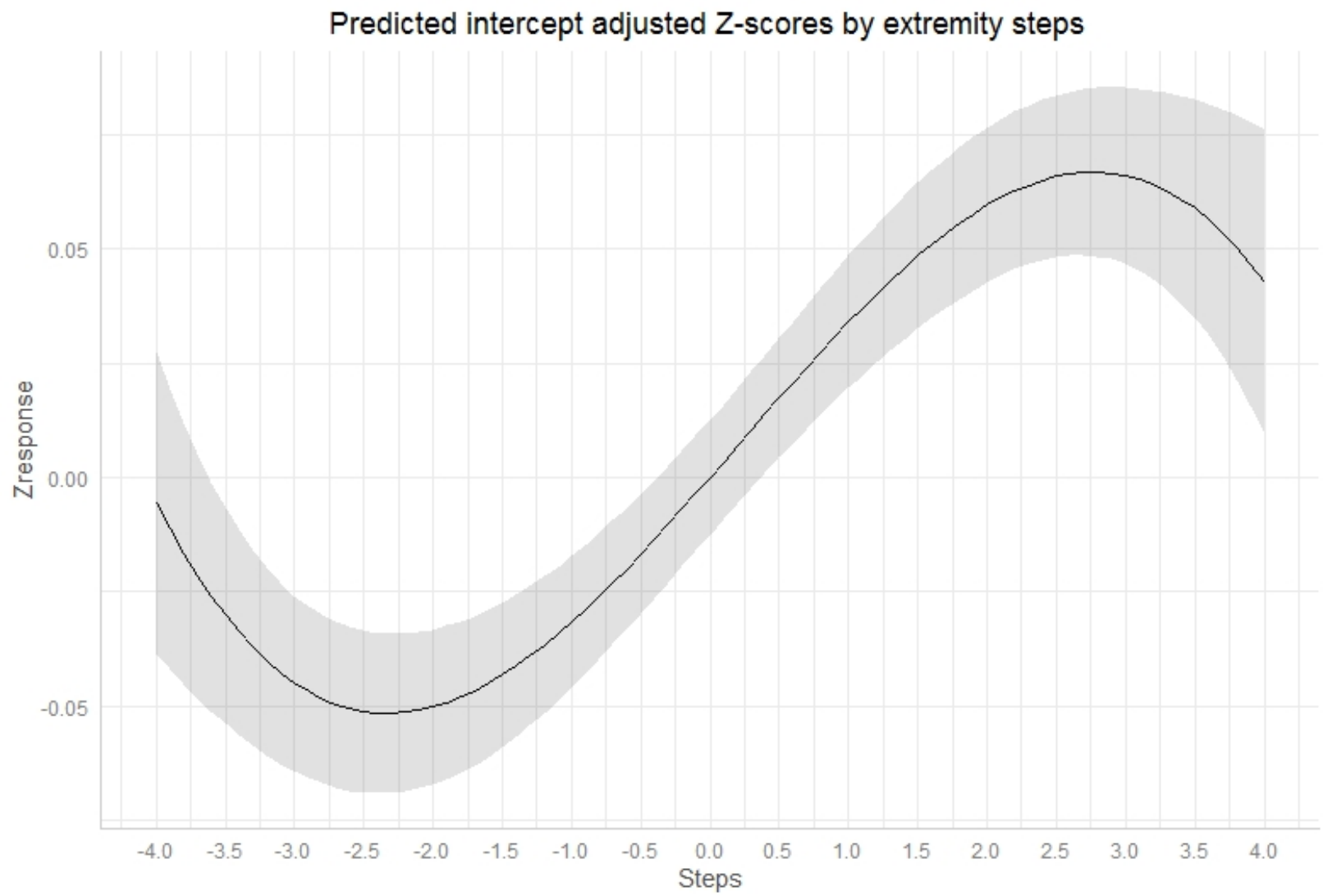

Figure 6. Predicted intercept adjusted marginal Z-scores for the trustworthiness dimension at each extremity step and predicted $95 \% \mathrm{CI}$

Despite the visual evidence against the presence of a turning point and the lack of convincing evidence for even a decrease in assimilative effects in the two lines test, the bootstrapping procedure detailed in Study 1a was conducted for this dataset as well to confirm 
the conclusions from the other analysis. In line with the evidence from the other analysis, all of the 3000 iterations returned no turning point for upward comparisons within the measured range of $4 S D, S E=0.006$, Bias $=-0.00,95 \% \mathrm{CI}[4,4]$ and only a limited few for downward ones, $-4 S D$, $S E=0.11$, Bias $=-0.07,95 \% \mathrm{CI}[4,-3.62]$, resulting in the window of assimilation spanning the entire range of measured steps, $8 S D, S E=0.11$, Bias $=-0.07,95 \%$ CI $[7.62,8]$. These results combined with the previous analysis suggest that only consistent assimilation occurs for this judgment.

\section{Discussion}

As was found in Barker et al. (2020), the current judgment showed only convincing evidence of increasing assimilation that levelled off for more extreme standards, but did not show significant signs of a reduction in strength, nor actual aggregated contrast effects. It is of course possible that even more extreme standards outside of this range might still produce consistent contrast effects, although broadening the possible window of assimilation even further would mean even fewer standards would exist in the population capable of eliciting the contrast effect making it decreasingly relevant in any real world scenario.

In any case, the marked shift in the dynamic pattern across the measured extremity spectrum indicates its sensitivity to the judgment that is being made, with no meaningful contrast

effects occurring in this context. Although there is some evidence for an upper limit to the size of the assimilation effect, it is unclear if this is due to the increased number of contrastive judgment or some other mechanism that limits the range of plausible values a participant may be willing to provide. In addition, it remains unclear if the increased assimilative effect for this judgment may reflect characteristics on the item level or could be a broader phenomenon for all judgments related to the Trustworthiness dimension. This question will be addressed in a later section, but 
first the sensitivity to judgment context will be shown to be able to produce the opposite pattern with only signs of consistent contrast effects for some judgments.

\section{Study 1c: Dominance}

In contrast to the text book pattern found for the Extraversion judgment and the purely assimilative effect for the Trustworthiness judgment, a surprising lack of assimilative effects were found in previous work for the facial dimension of dominance (Barker et al., 2020). Due to the limited and arbitrary selection of standard extremities in this previous work, it remains unclear whether this judgment has an exceptionally narrow window of assimilation or a complete lack of consistent assimilation effects altogether. Distinguishing these possibilities would be challenging without the very fine-grained manipulation of standard extremity that the extended CJT paradigm provides. Thus, this investigation will attempt to further investigate the extent of the contrastive variability that was found in the previous work for this judgment (i.e., "How many times does the target enforce his opinion in 6 months?").

\section{Method}

Participants. With similar power considerations as in Study 1b, 160 German speakers were recruited on campus at the University of Cologne to complete the study for a monetary reward of 10 euros. Two participants dropped out before completion, leaving a final sample of 158 participants, $38.6 \%$ of which were female and who had an age between 18 and 42 years $(M=$ 22.52, $S D=4.14)$

CJT. The task was again identical to Study $1 \mathrm{a}$ and $\mathrm{b}$ other than the open ended item that was now related to the dominance dimension and worded as "How many times does the target enforce his opinion in 6 months?" taken from Barker et al. (2020). 
Data treatment. The same data treatment was administered as in the previous studies. Trials were excluded from analyses if they were non-numeric or empty ( $4.3 \%$ of trials), or if they were preceded by a failed attention check $(11 \%)$. Al other trials were used to calculate $\mathrm{Z}$-scores per participant where possible after which they were truncated above 3 or below -3 as representing extreme values $(1.4 \%)$. Combined this meant that a total of $15.8 \%$ of all trials were not included in the final analyses with 7 participants not providing enough usable trials to be included in the analyses. The final sample used in the analyses thus consisted of 151 participants.

\section{Results}

The main analysis was identical to that used in studies $1 \mathrm{a}$ and $1 \mathrm{~b}$, utilizing a mixed models regression with REML estimation with fixed effects up to the third polynomial for the extremity steps, with identical but orthogonal and uncorrelated random slopes and intercepts for each participants. The cubic model showed a small significant negative third order term in line with the predictions, but this was not the case for the expected positive linear term, which in this dataset was negative and non-significant (Table 3). In Figure 7, one can see a lack of consistent assimilative effects for the moderate standards, but with slight contrast away for more extreme standards.

Table 3

All fixed effects and related statistics from mixed model analysis.

\begin{tabular}{llrrrrr}
\hline & $B[95 \% \mathrm{CI}]$ & $S e$ & $d f$ & $t$ \\
\hline Fixed effects: & & $-0.0030[-0.0146 ; 0.0087]$ & 0.0060 & 133.074 & -0.497 & .621 \\
& $\mathrm{x}$ & $-0.0018[-0.0041 ; 0.0005]$ & 0.0012 & 151.463 & -1.535 & .127 \\
& $\mathrm{x}^{2}$ & $-0.0008[-0.0016 ;-0.0001]$ & 0.0004 & 143.981 & -2.229 & .027 \\
& $\mathrm{x}^{3}$ & & & & & \\
& & & & & & \\
& Intercept & $-0.0230[-0.0388 ;-0.0072]$ & 0.0081 & 277.073 & .005 \\
\hline
\end{tabular}

To ensure the more restrictive cubic form of the function did not mask any weak assimilative effects at very small intervals, the more flexible adjusted two lines test was 
implemented again on a simplified unidirectional dataset. Results showed no evidence for an initial increase, but rather a non-significant negative slope up to $.7 S D, B=-0.03, Z=0.70, p=$ .482 , with significant signs of contrast for values beyond that point, $B=-0.02, Z=-5.51, p<$ .001 . These findings seem in line with the visual inspection of Figure 4, where no clear signs of assimilation present themselves, but where contrastive effects do increase with more extreme standards.

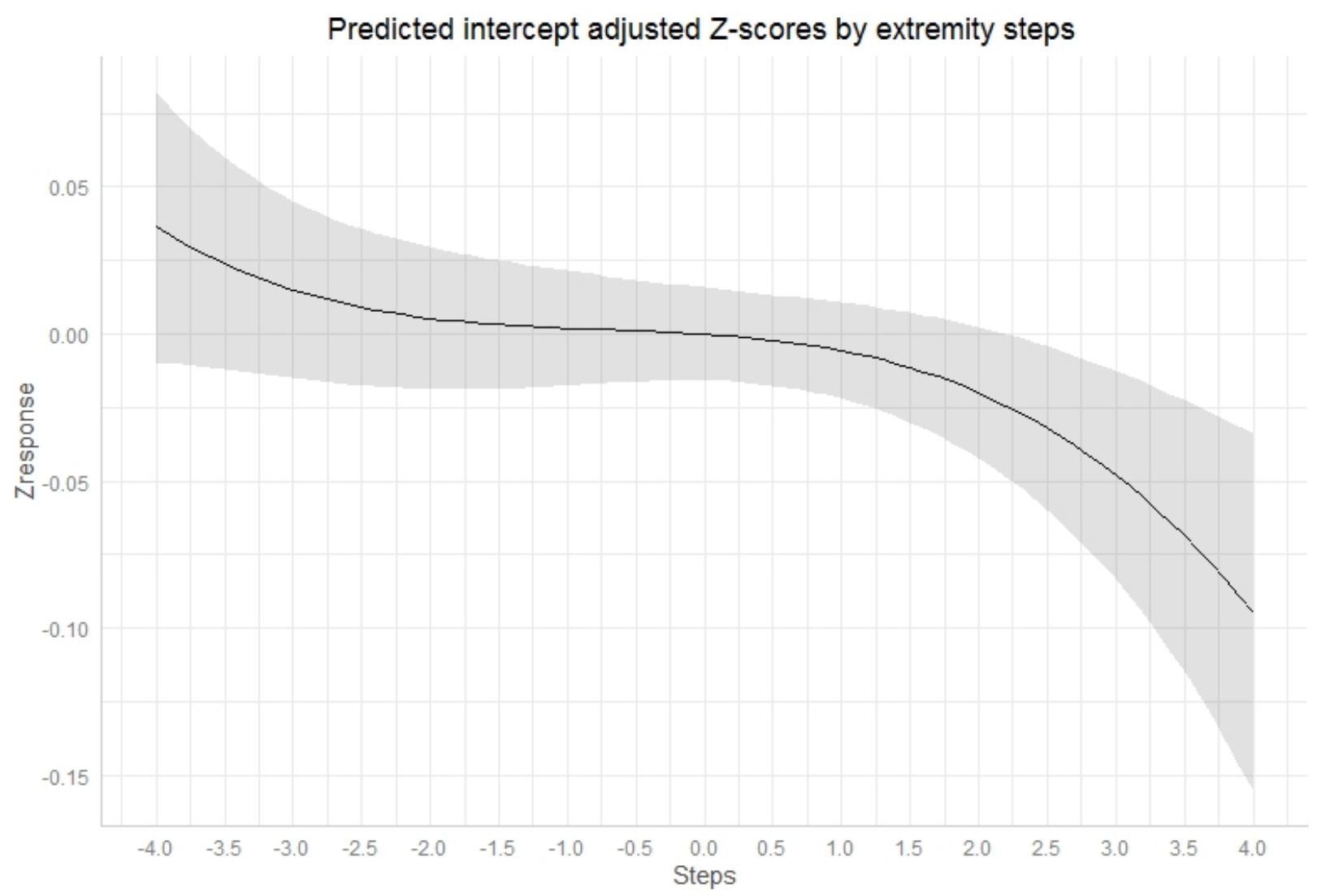

Figure 7. Predicted intercept adjusted marginal Z-scores for the dominance dimension at each extremity step and predicted $95 \% \mathrm{CI}$

Despite the lack of any evidence of assimilation, even for standards very close to neutral, a similar bootstrapping procedure as outlined in Study 1a was conducted to nevertheless attempt to estimate the turning point. Unsurprisingly, the large majority of the 3000 iterations returned an assimilative window of zero, $S E=1.25$, Bias $=0.64,95 \%$ CI $[0,3.85]$, meaning there were only 
signs of contrast in most cases for both upward, $0, S E=0.38$, Bias $=0.17,95 \%$ CI $[0,1.35]$ and downward comparisons, $0 S D, S E=0.90$, Bias $=-0.47,95 \%$ CI $[0,-2.71]$. In agreement with the expectations, it seems that there is no consistent assimilative tendency at any level for this specific judgment.

\section{Discussion}

In agreement with the findings in Barker et al. (2020) and in stark contrast to Studies 1a and $1 \mathrm{~b}$, no aggregated signs of assimilation were obtained across the extremity spectrum without a window of assimilation for even the narrowest ranges. Instead, no aggregated assimilative or contrastive effect seem to be present for the more moderate standards, whereas contrastive effects were found to increase towards the more extreme standards in a non-linear fashion. Again, the inability to disentangle assimilative and contrastive judgments leaves us strictly unable to clearly rule out whether this non-linearity is due to the absence of both assimilation and contrast for more moderate standards. However, a more parsimonious explanation in respect to the theory and other patterns presented previously may be that assimilation and contrast effects are merely in relative equilibrium even for these moderate standards due to an increased tendency for contrast brought on by the different judgment.

Although the variation uncovered here clearly shows the sensitivity of the dynamic interactive pattern to the context of the comparative judgment, it is still unclear if the variation found for these judgments, resides only at the item level or is indicative of dynamics at the level of the judgments themselves. This will be addressed in the next section.

\section{Studies 2a and 2b: Dimension level dynamics}

The last section clearly illustrates the highly sensitive nature of the dynamic interactive pattern to the exact judgment in which the comparisons are made. This is true to the extent that 
even completely contradicting conclusions can be drawn about the influence of social comparisons and the moderating role of standard extremity, ranging from purely assimilative to only contrastive, as well as both moderated by standard extremity. This poses a serious issue when interpreting and comparing results across studies that often include only one or two items (e.g. Ahrens, 1991; Buunk et al., 2007; Cash et al., 1983, Häfner \& Schubert, 2009; Martin et al., 2002; McFarland et al., 2001; Mendes et al., 2001; Raat et al., 2013; Smith \& Sachs, 1997), as it is impossible to assess whether these findings are specific to the exact judgment used in an investigation or if they truly reflect deeper theoretical mechanisms.

In addition, the issue may run deeper still as the findings so far cannot exclude the possibility that the variation could be directly linked to the judgment dimensions themselves rather than caused by item-level characteristics. Therefore, the current section will move past the item-level, to assess the variation or consistency of dynamic interactive patterns for judgment dimensions as a whole. For this, two additional studies will assess if a more consistent pattern with clear Windows of Assimilation will emerge at the dimensional level for Dominance and Trustworthiness when measured with multiple items.

As participants often idiosyncratically construe trait dimensions in a somewhat egocentric manner (Dunning et al., 1989; Dunning \& Hayes, 1996), these investigation opted to use a population based generative approach to the formation of items that aim to capture the dimensions as a whole. As in the previous section, a description of the methodological approach for procedure and the main task for both studies will be given here, prior to the study specific details that can be found in the separate sections.

CJT-41. In order to decrease strain on participants and make the procedure more suitable for online use, the extended CJT used in the previous section was reduced to include only 41 
trials per participants, creating the new CJT-41. This will help the recruitment of the large number of unique participants that will be necessary to accurately model the dimension level dynamics. To still conserve the same granularity with the reduced number of trials in the CJT-41, the exact steps of extremity that participants were exposed to was varied between respondents. For this, each participant was randomly assigned to one of two groups, the first of which judged 41 faces with standards that varied between $-4 S D$ to $+4 S D$ in intervals of $0.2 S D$. The second group judged faces for 40 steps of $0.2 S D$ starting at $-3.9 S D$ to $3.9 S D$ as well as the neutral $0 S D$ comparison. Combined the two groups thus provided measurement instances at each of the 81 steps that were also included in the extended CJT.

In addition to the shortening of the task, participants were now randomly assigned to answer one of 6 items for all trials in order to investigate the comparative pattern across items. Additionally, the attention check, which was mapped to keyboard buttons in the previous studies, was now completed by clicking a radio button underneath the images to identify the judgment target.

Stimuli. Eighty-one facial pairs similar to those used in the previous studies were created, with comparison standards at each of the $0.1 S D$ steps between $-4 S D$ and $4 S D$. The dimensions of the stimuli were again 300 by 300 pixels and displayed in the same way as in the previous studies. However, as the study was conducted online the size on screen, viewing angles and viewing distances were different between participants.

To develop the range of items needed for the studies in a more data driven fashion, a twophased pre-test was conducted for each dimension. The first part consisted of asking an online sample of 50 participants to provide 5 examples of particularly dominant/trustworthy behaviors and 5 examples of submissive/untrustworthy behavior in an open ended fashion. The responses 
were then processed into a list of the most frequently mentioned examples. The 10 most commonly given examples of dominant/trustworthy and 10 most common submissive/untrustworthy behaviors were then used in the second phase where a new sample of 180 participants made a single judgment for every item about an isolated face which randomly varied along the dominance/trustworthiness dimension in $1 S D$ steps between $-4 S D$ and $4 S D$. This resulted in approximately 20 measurement instances at each step for each item. The resulting data were then included in a mixed models linear regression for each item separately. The three items that showed the strongest linear relationship for submissive/trustworthy behavior were selected. Furthermore, the items used in Study $1 \mathrm{~b}$ and 1c were included again along with the top two new items for dominance/untrustworthiness, see Table 1A and 2A in Appendix $\mathrm{C}$ for a list of all the selected items. A more in depth description of the pre-testing phase can be found in the supplemental materials.

Additional measures. Besides the basic demographics of age, sex, and education, an additional item at the start of the survey was included asking participants to describe what was presented on an image in order to make sure the images were indeed loading correctly. Furthermore, an item was included after the main CJT-41 posing the same question participants had been asked throughout, but this time they were to use themselves as the judgment target. Both these items were not used in any of the analyses, but their data are included in the supplemental materials.

Finally, a new data-quality item was included at the end of the study which allowed participants to report if their responses were made in a conscientious manner and the resulting data should be used or not to increase the quality of the data in the final analyses. Responses to this item were guaranteed not to negatively affect participants or their compensation in any way, 
but was merely to help clean up the data for use. Responses to this item ranged from "Definitely do not use my data" (1) to "Definitely use my data" (4). Any responses of 2 or lower were used to exclude participants from the analyses.

\section{Study 2a}

In this pre-registered study (https://aspredicted.org/9h8tj.pdf) the dynamic interactive pattern of the dominance dimension as a whole was investigated using multiple participant generated items. The pre-registration was followed in full unless explicitly stated otherwise.

\section{Method}

Participants. Using simulations, a sample size of 800 participants was determined to be sufficient to find effects of a similar size to those found in a pilot test of the paradigm using only 41 trials and the single extraversion judgment with some room for drop out $80 \%$ of the time. ${ }^{4}$ An online sample of 800 U.S. based MTurk workers were recruited for a monetary compensation of $\$ 1.20$. Nine participants completed the study without claiming their compensation and thus were not accounted for in the quota of 800 , meaning the total sample of completed responses consisted of 809 participants. This final sample was $53.8 \%$ female and was aged between 20 and 96 years $(M=37.62, S D=11.73)$

CJT-41. As explained in more detail in the introductory section for Study $2 \mathrm{a}-\mathrm{b}$, participants will be presented with 41 pairs of facial images each. Participants will be asked to judge a neutral target face on one of six open ended items pretested to relate to the dominance dimension, see Table 1A in Appendix C. The second face in the pair will not itself be judged but

\footnotetext{
${ }^{4}$ Findings for this pilot test will not be discussed here in detail to conserve space, but the pattern found in Study 1a was replicated successfully, with a significant linear, $t(1055.44)=2.50, B=0.0156, p=.013$, and cubic effect, $t(748.80)=-3.96, B=-0.0023, p<.001$, and a turning point around 2.61SD. A more detailed description can be found in the supplemental materials.
} 
will be the standard ranging from $+/-4 S D$ away from the neutral target in $0.1 S D$ steps on the dominance dimension. Participants will only be exposed to half of the resulting 81 pairs, resulting in 41 trials in total.

Data treatment. As in all previous studies, trials with non-numeric or empty responses were removed $(0.2 \%)$ as were those in which the attention check was failed (4.8\%). Responses were then used to calculate z-scores per participant and truncated above 3 or below $-3(2.2 \%)$. For the three reversed items, the z-scores were flipped so that higher scores always reflected more dominant behaviors. At least one of these exclusion criteria was met in $7.3 \%$ of all trials resulting in twelve participants not providing any usable trials. Finally, the new data-quality item was used to further exclude 26 participants who indicated they did not think their data were of high enough quality to be included (a score of 2 or lower), leaving 771 participants in the dataset for use in the analyses.

\section{Results}

Once again, the initial analysis consisted of a mixed models regression using a REML estimation, but considering the reduced number of measuring points a different model structure was used. As the first and third order polynomial terms for the extremity steps are of most theoretical relevance for detecting assimilation and contrast and there were no signs of a meaningful quadratic term in the previous studies, along with recent meta-analytical evidence showing no differences between upward and downward comparisons (Gerber et al., 2018), only these two terms were pre-registered to be included as fixed effects in the model. As a result the model will no longer be able to distinguish between upward and downward comparisons and, thus we will also not estimate the turning point for upward and downward comparisons separately as these will necessarily be fully symmetric under the models constraints. The full 
random slope structure and intercepts were fitted per participant with only random intercepts included per item, as the data were not sufficient to support the addition of the linear and cubic effects for each item. ${ }^{5}$ Note, that this entails that the results related to the linear and cubic terms cannot strictly be generalized to the judgment dimension as a whole beyond the items that were used in this investigation.

In contrast to the results found in Study $1 \mathrm{c}$ that used a single judgment, the data from this study did show evidence for assimilation for the dominance dimension as a whole as reflected in a significant first order effect, as well as for contrast seen in the negative third order terms, see Table 4, which together describe the hypothesized the S-shaped curve, see Figure 8.

Table 4 All fixed effects and related statistics from mixed model analysis.

\begin{tabular}{|c|c|c|c|c|c|}
\hline & $B[95 \% \mathrm{CI}]$ & $S E$ & $d f$ & $t$ & $p$ \\
\hline \multicolumn{6}{|l|}{ Fixed effects: } \\
\hline $\mathrm{x}$ & $0.0155[0.0036 ; 0.0274]$ & 0.0061 & 1097.68 & 2.55 & .011 \\
\hline$x^{3}$ & $-0.0020[-0.0031 ;-0.0009]$ & 0.0006 & 774.38 & -3.62 & $<.001$ \\
\hline Intercept & $-0.0001[-0.0263 ; 0.0265]$ & 0.0053 & 5.02 & 0.01 & .994 \\
\hline
\end{tabular}

Additional analyses were again performed to formally test whether the descriptive Sshaped curve includes both a significant initial increase and subsequent decrease of scores respectively. A simplified unidirectional dataset was, therefore, used in the adjusted two lines procedure with random intercepts for participants only. Both the linear increase up to $1.1 S D, B=$ $0.06, Z=2.18, p=.03$, and the decrease thereafter, $B=-0.03, Z=-3.56, p<.001$, were found to be present and significant in these data.

\footnotetext{
${ }^{5}$ The random effects structure used in the final model diverges from the one described in the pre-registered model, which only mentions linear random effects and intercepts for participants and no random intercept for each item. The decision to include these extra random effects was made to increase the generalizability of the model after it became clear that the data could support this more complex structure. Results using the simplified structure from the preregistered analyses are almost identical and do not change the conclusions.
} 


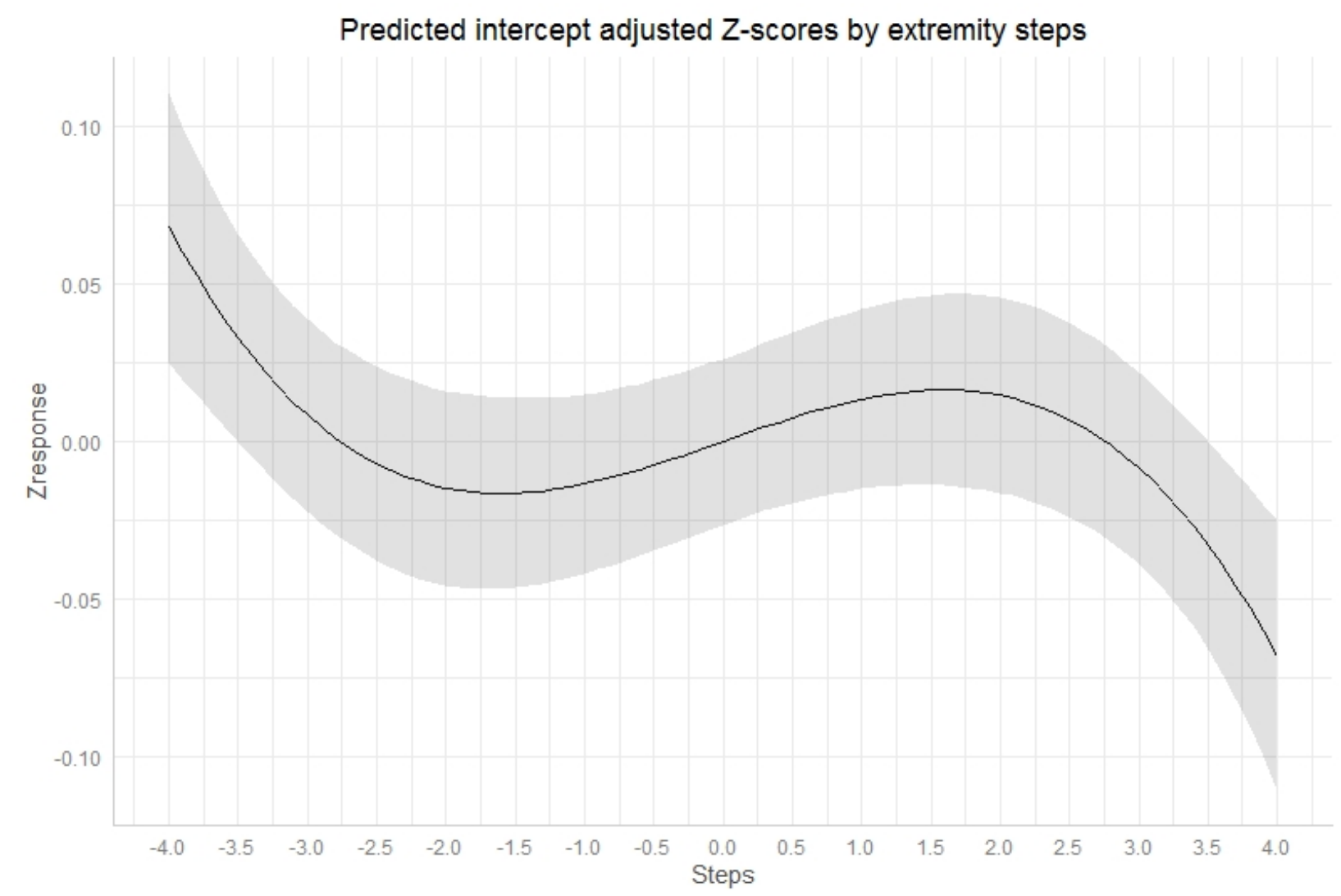

Figure 8. Predicted intercept adjusted marginal Z-scores for the dominance dimension at each extremity step and predicted $95 \% \mathrm{CI}$

Finally, the bootstrapping procedure described in study la was implemented. The results after 3000 iterations showed the average turning point for the judgments was around 2.76SD, SE $=0.34$, Bias $=-0.05,95 \%$ CI $[1.99,3.14]$, with the window of assimilation spanning 5.52SD, SE $=0.68$, Bias $=-0.10,95 \%$ CI $[3.98,6.27]$. Taken together the results suggest that the consistent contrast found in Study 1 is indeed a reflection of item level rather than dimension level heterogeneity in comparison patterns.

\section{Discussion}


In contrast to the results from Study 1c, clear assimilation and contrast effects emerged for the dominance dimension when measured across the participant generated items, providing support for the theoretically predicted comparative pattern of aggregated assimilation to moderate and contrast from more extreme standards. In addition, these findings suggest that the previously found increase in contrastive strength is likely caused by item level variables, rather than related to the judgment dimension of dominance as a whole.

The dynamic interactive pattern that was uncovered for this dimension has, furthermore, provided stronger estimates of what may constitute an extreme standard for facial dimensions. Under the current conditions, standards which are more extreme than those at the turning point of 2.76SD seem to be able to produce consistent contrast. However, these guidelines might vary significantly between judgment dimensions, which is especially likely for the dimension of trustworthiness, which in Study $1 \mathrm{~b}$ showed only signs of assimilative effects. Therefore, the next study will aim to offer a similar dimension level account of the comparative pattern for Trustworthiness using a number of participant generated items.

\section{Study 2b}

The results from Study 2 a make it apparent that item level variation can strongly influence the comparison dynamic. It is still to be determined whether such item level effects also underlie the purely assimilative results found in Study $1 \mathrm{~b}$ for the trustworthiness dimension. The current study, therefore, investigated the comparison dynamic for the trustworthiness dimension using multiple items in a pre-registered study (https://aspredicted.org/zc3at.pdf).

\section{Method}


Participants. As in Study 4, 800 participants were sought on the online platform MTurk for a $\$ 1.20$ reward. The recruited sample was $45.6 \%$ female and was aged between 19 and 73 years $(M=36.01, S D=10.46)$.

CJT-41. The task remained the same as in study $2 \mathrm{a}$, with the exception of the six items that now were pretested to relate to the trustworthiness dimension, see Table 2A in Appendix C.

Data treatment. Data treatment was again in line with the procedure used in all the previous studies. Trials were removed if non-numeric or empty responses were given (4.2\%) and if the attention check was failed (8.1\%). The remaining scores were $\mathrm{z}$-transformed per participant where possible, with values above $3 S D$ or below $-3 S D$ being removed $(3.5 \%)$ after which zscores for the three reverse coded items were flipped. A total of $15.6 \%$ of all trials were deemed non-informative by these exclusion criteria, which meant no high quality trials remained for 25 participants. Another 26 participants were removed as they themselves indicated their data was of low quality, leaving 749 participants to be analyzed.

\section{Results}

A mixed models regression using a REML estimation with the first and third order polynomial terms for extremity were once again included as fixed effects with similar orthogonal uncorrelated random slopes and intercepts for participants in the main analysis. In addition, random intercepts were included for each item, see Footnote 3. As with the previous investigation of this dimension, the model showed both a significant positive first order and negative third order effects (Table 5). However, this time the curve did seem to cross the 0-point within the measured range suggesting contrast may have occurred (Figure 9). 
Table 5

All fixed effects and related statistics from mixed model analysis

\begin{tabular}{|c|c|c|c|c|c|}
\hline & $B[95 \% \mathrm{CI}]$ & $S E$ & $d f$ & $t$ & $p$ \\
\hline \multicolumn{6}{|l|}{ Fixed effects: } \\
\hline $\mathrm{x}$ & $0.0376[0.0244 ; 0.0508]$ & 0.0067 & 1079.73 & 5.59 & $<.001$ \\
\hline$x^{3}$ & $-0.0040[-0.0052 ;-0.0028]$ & 0.0006 & 735.32 & -6.53 & $<.001$ \\
\hline Intercept & $-0.0021[-0.0168 ; 0.0205]$ & 0.0095 & 4.00 & 0.19 & .854 \\
\hline
\end{tabular}

The adjusted two lines procedure was again implemented as an additional analysis of the increase and decrease of the S-shaped curve separately. The assimilative linear increase was found to be significant up to $2.1 S D, B=0.03, Z=2.28, p=.02$, as well as a decrease for more extreme values, $B=-0.07, Z=-4.60, p<.001$, which is in support of a pattern of assimilation followed by contrast as extremity increases.

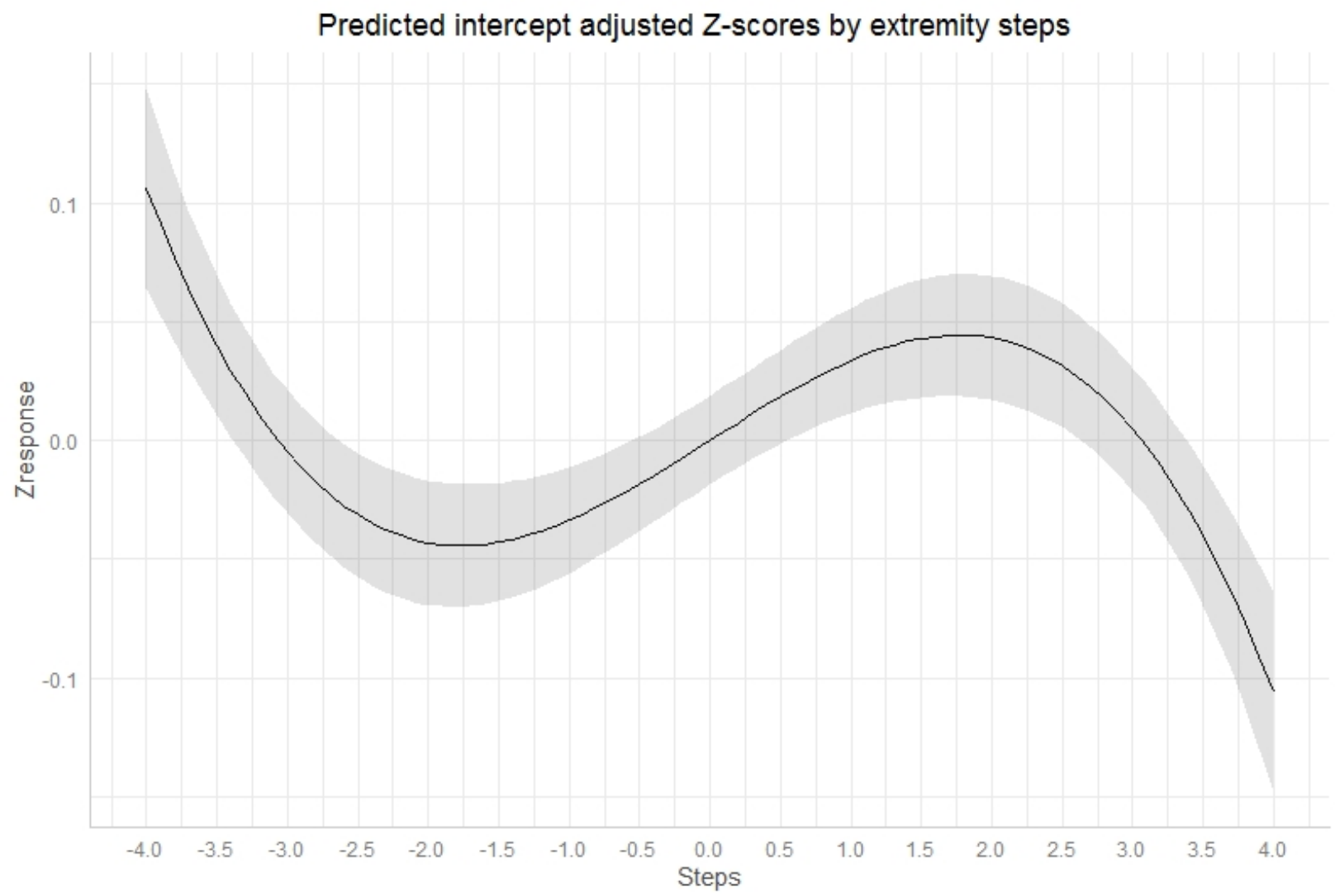

Figure 9. Predicted intercept adjusted marginal Z-scores for the trustworthiness dimension at each extremity step and predicted $95 \% \mathrm{CI}$ 
Lastly, the bootstrapping procedure was implemented again with 3000 iterations. The results this time did show a clear turning point of around $3.06 S D, S E=0.10$, Bias $=-0.01,95 \% \mathrm{CI}$ $[2.84,3.25]$, with the widow of assimilation spanning $6.12 S D, S E=0.20$, Bias $=-0.0195 \% \mathrm{CI}$ $[5.68,6.50]$, which is surprisingly close to the turning point found for the Dominance dimension in Study 3a. Taken together the results seem to be firmly in line with the theoretical prediction, meaning the variation found in earlier studies was once again likely restricted to item level effects.

\section{Discussion}

The purely assimilative patterns found in Study $1 \mathrm{~b}$ seem again to be related to the item being considered rather than that of the judgment dimension itself, as was the case for the contrastive findings for the dominance dimensions. Across multiple items, consistent assimilation and contrast effect were found at the aggregated level depending on the extremity of the presented standard. These results further highlight the substantial influence item formulations can have on the dynamics of the comparison process, but also highlight a relative consistency that can be found at the level of judgment dimensions. Indeed, the uncovered pattern showed a very similar boundary to the assimilative effect as was found for the dominance dimension, with assimilative effects this time disappearing when standards around 3.06SD or higher were present. Taken together with the findings found for the dominance dimension, we may offer a first rule of thumb for future research that extreme standards, for facial judgments at least, are those that exceed $3 S D$.

Regretfully, the current work cannot speak towards the exact item level conditions that are responsible for the purely contrastive effects that presented themselves in Study 1c or purely assimilative ones in Study 1b. However, it may be that while the initial extraversion item 
referred to a behavior that does not require a third social entity (i.e., 'How often does the target go out in 6 months?'), the trustworthiness item does (i.e., 'How many times does the target deceive somebody in 6 months?'). This social aspect may have highlighted the potential social relation and the way in which both can influenced to think in similar ways by their surroundings. On the other hand, the dominance item (i.e., 'How many times does the target enforce his opinion in 6 months?') could have evoked an idea of "enforcing one's opinion" over the comparison standard as only one of the two can get their way within a pair. This, in turn, could have led to an increased emphasis on the differing level of dominance between the stimuli and contrast effects.

However, due to the large variation in the items and their linguistic context it is not possible to offer a satisfactory conclusion regarding the precise mechanisms that caused these differencing comparative patterns. Therefore, until future research offers clear guidance on the development of comparative judgments, research in this area should steer clear of using single or handpicked items, opting instead to use multiple items and analyzing theory at the level of the judgment dimensions.

\section{Studies 3a, 3b and 4: A Holistic Framework for Comparative Research}

Up to this point the current investigation has merely uncovered the dynamic interactive pattern and its sensitivity to item level variation addressing two major shortcomings in the existing literature. However, the current approach can also offer a framework to advance theory beyond these basic issues of study design, by offering stronger and more precise tests of other theoretically influential variables. Using the CJT-41, or a similar approach that includes multiple standards across the extremity spectrum, offers the unique ability to clearly distinguish the attenuation of comparative effect from a situation where both comparative outcomes are in 
equilibrium. Indeed, these phantom comparison effects are clearly visible in the dynamic patterns uncovered in the last section, where they form the turning points where aggregated assimilation becomes contrast. The current section will illustrate how more narrow approaches may incorrectly confuse these phantom comparisons with true attenuation in comparative effects using the CJT-41. To do this, it will show the unique shifts in the dynamic pattern of the trustworthiness dimension that is brought on by two distinct moderators, a focus on similarities rather than differences (Mussweiler, 2001) and the presence of differing category information (Mussweiler \& Bodenhausen, 2002).

\section{Study 3a}

This first investigation will focus on the theorized shift in the dynamic interactive pattern of assimilation and contrast for the trustworthiness dimension brought on by a focus on differences rather than similarities. It will again employ the CJT-41, but this time it will model and compare the two separate curves under each focus in a pre-registered study (https://aspredicted.org/tk46j.pdf). Although the typical procedure for inducing different comparative foci has been to include a procedural priming task in which a focus is given to finding similarities or differences prior to the focal judgment (Mussweiler, 2001), these subtle priming effects might not remain influential across the many trials that are necessary to model the dynamic interactive pattern accurately ${ }^{6}$. Therefore, the current work will focus on explicit instructions in every trial to focus on similarities or differences prior to making a judgment to allow for consistent manipulation at each point.

\footnotetext{
${ }^{6}$ An additional study was conducted based on the findings described here using the procedural priming manipulation by Mussweiler (2001), but remained inconclusive due to the high sensitivity to the repeated measures design. A summary and the dataset are again available on the OSF page.
} 
In general, a positive linear effect across these comparisons would again be expected as a sign of aggregated assimilation, with a negative cubic term indicating an increase in contrastive judgments for more extreme standards. We expect the focus condition to influence these two vital estimates in a way in which assimilative judgments are reduced under a dissimilarity focus compared to under a similarity focus. In the broadest terms this should be reflected in a significant increase in fit when including the condition variable and its interactions in the model. To test the more specific expectations that the condition will reduce the overall strength of assimilation and lead to consistent contrast effects for more moderate standards, the area of assimilation (AoA) and the window of assimilation (WoA) will be estimated using a bootstrapping procedure. A smaller AoA and narrower WoA are expected in the dissimilarity compared to the similarity focus condition.

\section{Methods}

Participants. Simulations revealed that, based on the parameters found for the Trustworthiness dimension in Study 2b, 950 participants would be necessary to find a difference between the two conditions at least $80 \%$ of the time if the linear effect changes by $B=.018$. Therefore, a total of 950 US-based MTurk participants were recruited to complete the study for a reward of $\$ 1.20$. The final sample in this study was $51.4 \%$ female and was aged between 18 and 76 years $(M=37.14, S D=11.84)$.

CJT-41. The CJT-41 was administered in the exact same way as in Study $2 \mathrm{~b}$ focusing on the trustworthiness dimension. This time, however, half of the participants will be explicitly asked in each trial to focus on similarities, with the other half focusing on differences, during the judgments. 
Additional measures. Age, sex, and education were recorded as basic demographics. At the start of the survey, an item asked participants to describe what was presented on an image depicting shapes and colors, to ensure images were displayed correctly in the majority of cases. In addition, an item after the main task required participants to indicate whether they were asked to focus on similarities or differences throughout the trials, which acted as a simple manipulation check and exclusion criterion if answered incorrectly. Finally, the self-reported data-quality item at the end of the study asked participants if their data should or should not be used based on their effort throughout the task.

Procedure. Participants were recruited using the online platform MTurk and fully informed about the data storage policy, procedure, and their rights before they were asked for their consent to take part in the study. Participants first responded to the image identification question, followed by the basic demographics. Participants were then randomly allocated to a similarity condition, where they were instructed to look at both images and focus on similarities or differences before making their final judgment. The CJT-41 was then explained in detail and two practice trials were presented before the main batch of 41 trials started. Throughout the trials participants were reminded in the middle of each page to focus on similarities or differences respectively. Upon completion of the main task, participants were presented with the manipulation check question followed by the data-quality item. Finally they were debriefed, thanked and given their compensation.

Data treatment. As pre-registered, all trials with non-numeric or empty responses were removed (4.3\%) as well as any responses marked by a failed attention check $(6.7 \%)$. The remaining responses were z-transformed per participant where possible to ensure similar responses scales across items and respondents. The resulting scores were then truncated above 3 
or below $-3(3.8 \%)$ and flipped for reverse coded items so that higher scores reflect more Trustworthy behaviors. Combined these criteria resulted in $14.7 \%$ of all trials being removed. Lastly, a large group of 162 participants failed to correctly identify the condition they were assigned to, while 41 indicated their data should not be used. These pre-registered criteria combined lead to the exclusion of 216 participants, which was a far larger amount than expected. The final sample that could be used in the analyses, thus, only included 734 participants.

\section{Results}

The pre-registered analyses consisted of running two mixed models regressions using maximum likelihood (ML) estimation with the lme4 package (Bates et al., 2015) to allow for model comparison. Both models included linear and cubic fixed effects for the extremity steps, and had a similar structure of orthogonal uncorrelated random slopes and intercepts for participants and items. The full model additionally included fixed effects for the condition variable and all interactions, estimating the marginal effect of the focus condition on the shape of the curve ${ }^{7}$. Finally, an analysis of deviance was used to compare the full model to the model without any effect of condition on the comparison pattern. The results showed that, while the Sshaped curve in the difference focus condition was descriptively more shallow in line with the expectations (Figure 10), this difference failed to reach the standard level of significance in these data, $\chi^{2}(3)=7.17, p=.067$, providing no convincing evidence for the moderating influence of the comparative foci on the comparison pattern overall.

\footnotetext{
${ }^{7}$ The random effects structure is more extensive than the one described in the pre-registration. Upon reviewing the data, it was found that this more extensive random effects structure was able to be modeled, which is preferable since it allows for broader generalizability of the results. The simplified structure from the preregistered analyses only increase the strength of the uncovered associations.
} 
Although the focal test failed to reach significance, separate models per condition were fit using restricted maximum likelihoods (REML) to gain some indication of their separate comparative patterns and estimate the differences in area of assimilation and the window of assimilation. These analyses showed that in the similarity focus condition, both the linear and cubic effects were significant and in the direction expected for a pattern of assimilation to moderate and contrast from extreme standards (Table 6). However, in the difference focus condition, the positive linear effect was not detected, but the negative cubic effect was still present (Table 7) providing only evidence for contrast under a dissimilarity focus.

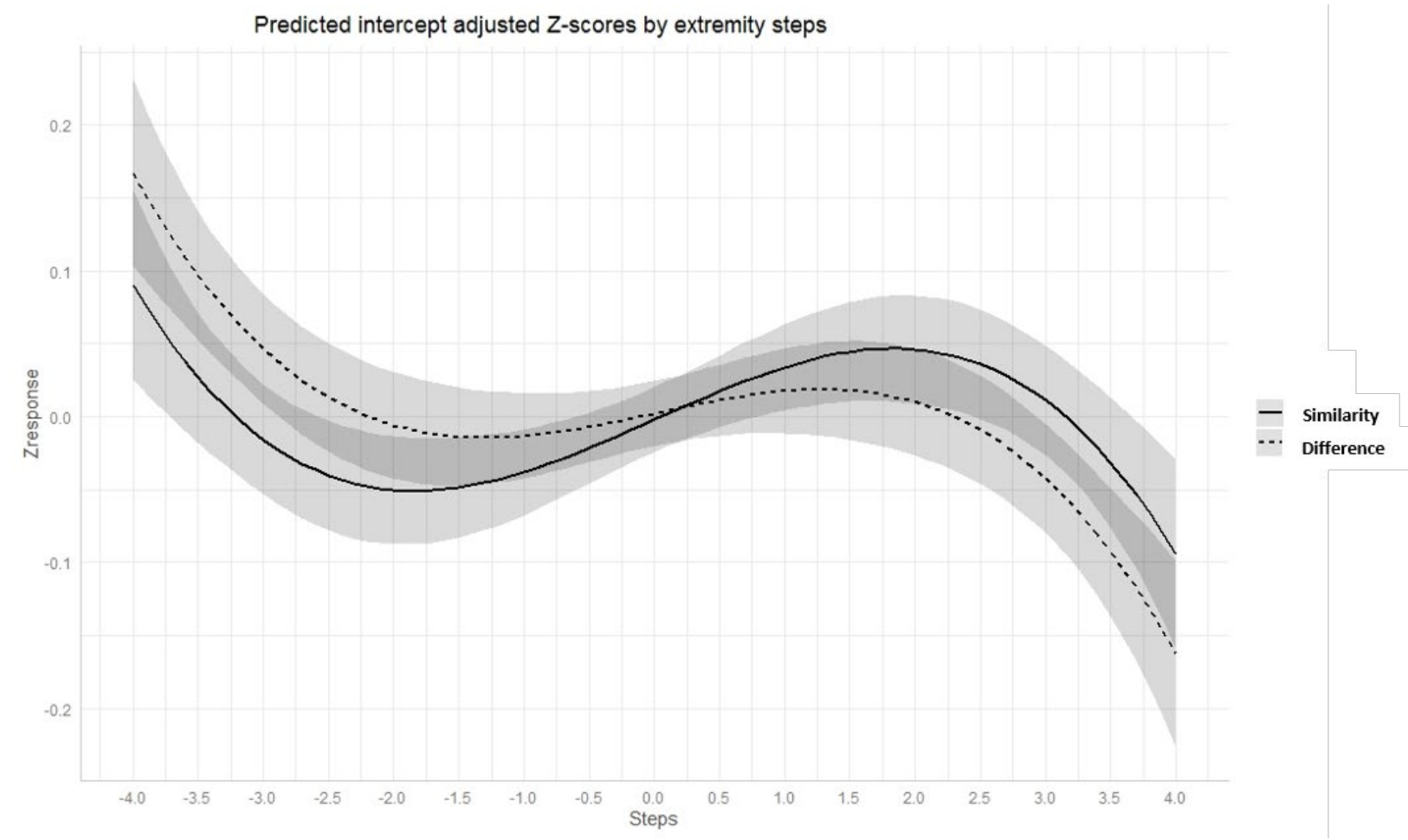

Figure 10. Predicted intercept adjusted marginal z-scores for the trustworthiness dimension separately for the different focus conditions at each extremity step and their predicted $95 \% \mathrm{CI}$ (created with the ggeffects package, Lüdecke, 2018)

To estimate the descriptive statistics of the area of assimilation and windows of assimilation for each condition, as well as their respective non-parametric percentile confidence intervals, the bootstrapping procedure was again implemented using a simplified model with 
maximum likelihood estimation and only random intercepts and linear slopes for participants and items. To calculate the AoA, the definite integral between zero and the upward turning point was returned. The WoA was found by subtracting the downward turning point from the upward one. Results showed that the AoA, the cumulative strength of assimilation, was larger under the similarity focus condition, $0.10 S D^{2}, S E=0.02$, Bias $<-0.001,95 \%$ CI $[0.06,0.15]$, than the dissimilarity condition, $0.03 S D^{2}, S E=0.02$, Bias $=0.002,95 \%$ CI $[0.00,0.06]$, but with percentile confidence intervals which overlapped in agreement with the main analysis. The WoA, indicating the range in which assimilation is more likely than contrast, was also found to be larger under a similarity focus, $6.35 S D, S E=0.28$, Bias $=-0.02,95 \% \mathrm{CI}[5.77,6.90]$, than a dissimilarity focus, $4.52 S D, S E=0.82$, Bias $=-0.18,95 \%$ CI $[2.16,5.33]$, with percentile confidence intervals that this time did not overlap.

Table 6.

All fixed effects and related statistics from mixed model analysis for similarity focus.

\begin{tabular}{|c|c|c|c|c|c|}
\hline & $B[95 \% \mathrm{CI}]$ & $S E$ & $d f$ & $t$ & $p$ \\
\hline \multicolumn{6}{|l|}{ Fixed effects: } \\
\hline $\mathrm{x}$ & $0.0401[0.0189 ; 0.0613]$ & 0.0108 & 7.23 & 3.71 & .007 \\
\hline $\mathrm{x}^{3}$ & $-0.0040[-0.0059 ;-0.0021]$ & 0.0010 & 4.39 & -4.10 & .012 \\
\hline Intercept & $0.0039[-0.0177 ; 0.0000]$ & 0.0110 & 4.80 & 0.36 & .737 \\
\hline
\end{tabular}

Table 7 . All fixed effects and related statistics from mixed model analysis dissimilarity focus.

\begin{tabular}{|c|c|c|c|c|c|}
\hline & $B[95 \% \mathrm{CI}]$ & $S E$ & $d f$ & $t$ & $p$ \\
\hline \multicolumn{6}{|l|}{ Fixed effects: } \\
\hline $\mathrm{x}$ & $0.0191[-0.0022 ; 0.0404]$ & 0.0109 & 7.70 & 1.76 & .118 \\
\hline$x^{3}$ & $-0.0038[-0.0057 ;-0.0019]$ & 0.0010 & 4.69 & -3.86 & .013 \\
\hline Intercept & $0.0084[-0.0137 ; 0.0305]$ & 0.0113 & 5.22 & 0.74 & .489 \\
\hline
\end{tabular}




\section{Discussion}

In this first investigation of the effect of different comparative foci on the dynamic interactive pattern of assimilation and contrast, the hypothesized effect did not reach the standard level of significance providing insufficient evidence to determine that there was an influence. However, the descriptive pattern and follow up bootstrapping tests did yield some evidence that the condition affected the window of assimilation specifically. Considering these conflicting data points a replication attempt was performed to provide more conclusive findings.

One may take note of the relatively low data quality that was achieved from the online platform, with $22.7 \%$ of respondents meeting the pre-registered exclusion criteria severely reducing the power to detect the expected effect. Therefore, the next study will be a close replication, but using the alternative platform Prolific.

\section{Study 3b}

This study will be a close replication of the previous one, with only the online platform used for recruiting participants being changed from MTurk to Prolific, which has been found to have a more diverse and honest population with comparable quality (Peer et al., 2017). As no changes were made to the general method or protocol, these sections will not be discussed here. Similarly the hypotheses, expectations and analyses for this study remain the same as in the previous study.

\section{Method}

Participants. A sample of 950 participants was this time sought on the Prolific platform for a slightly higher reward of $£ 1.50$ in an attempt to increase data quality. Five additional participant completed the study, but were not tallied by the platform resulting in 955 finished 
responses in total. This final sample consisted of $59.6 \%$ females and ranged from 18 and 82 years of age $(M=36.68, S D=12.06)$.

Data treatment. As in the previous study, non-numeric or empty responses were removed (3.6\%) along with trials in which the attention check was failed $(2.1 \%)$. Z-scores per participant were then created using the remaining responses where possible and truncated above 3 or below -3 (4.9\%), with scores flipped for reverse coded items. All together these criteria resulted in $10.7 \%$ of trials being excluded from the analyses. Sixty-eight participants did not correctly identify the condition they were assigned to and were removed, as well as 25 participants with low self-reported data quality. In total, all criteria combined called for the removal of 132 participants, resulting in a final sample of 823 to be used in the analyses. One might take note of the improved data quality using this alternative platform, with $13.8 \%$ of participants not providing usable data compared to the $22.7 \%$ in the previous study.

\section{Results}

Analyses identical to the ones used in Study 3a were performed on these new data. Results showed that the similarity focus condition this time was shown to have a significant influence on the comparison pattern, $\chi^{2}(3)=16.75, p<.001$, with the resulting curves descriptively again agreeing with the expectations that a focus on dissimilarities would lead to a more shallow curve, and seemingly narrower window of assimilation than a focus on differences (Figure 11). 


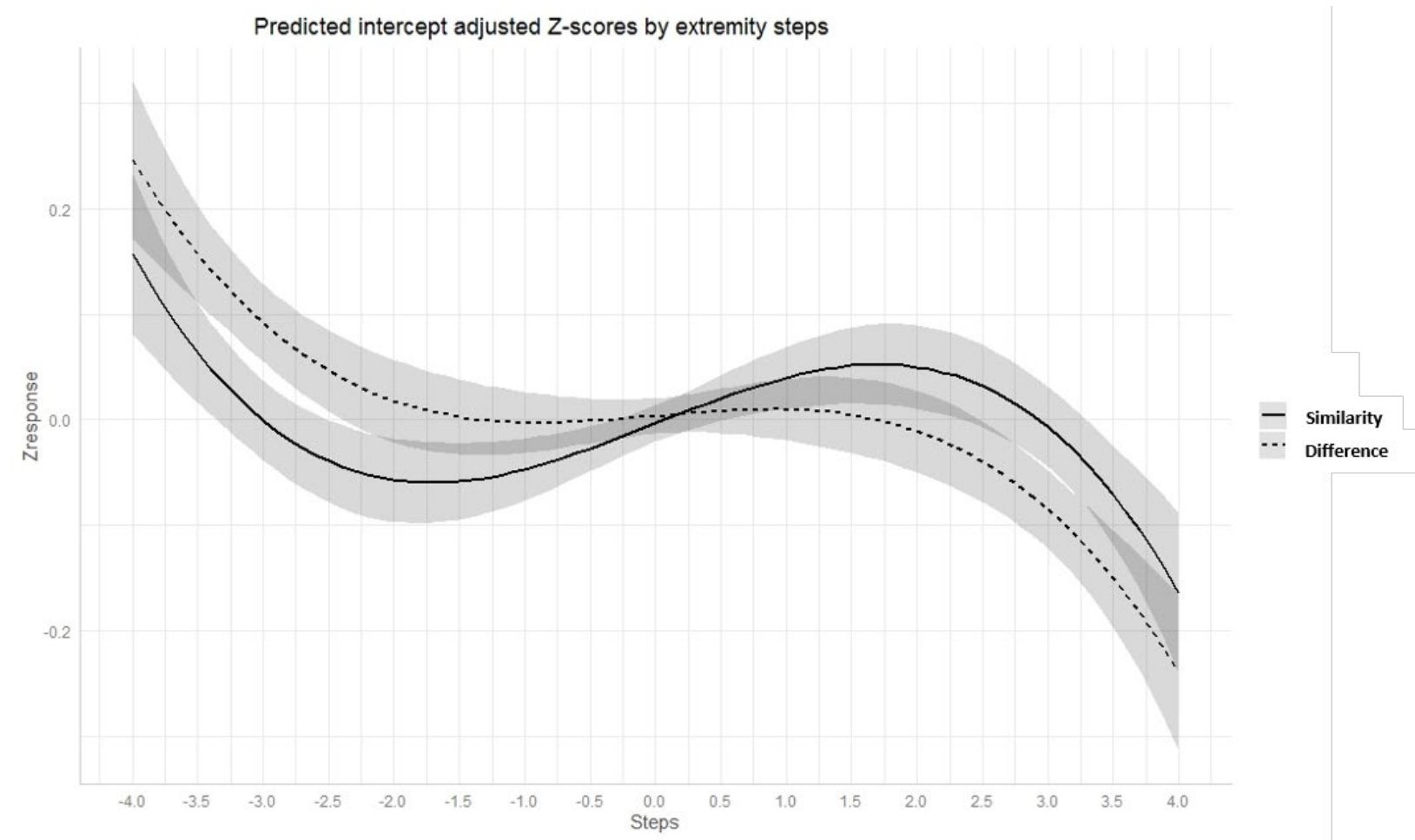

Figure 11. Predicted intercept adjusted marginal z-scores for the trustworthiness dimension separately for the different focus conditions at each extremity step and their predicted $95 \% \mathrm{CI}$

Separate mixed models analyses using REML were then conducted to gain an indication of the pattern in both conditions separately. Results showed the positive linear and negative cubic effects to be significant in the similarity focus condition (Table 8), indicating both assimilation and contrast had likely occurred. On the contrary, the difference focus condition again did not show a significant linear effect, but did show a significant negative cubic effect (Table 9), thus only providing clear evidence for contrast effects for increasingly extreme standards.

Table 8. All fixed effects and related statistics from mixed model analysis for similarity focus.

\begin{tabular}{|c|c|c|c|c|c|}
\hline & $B[95 \% \mathrm{CI}]$ & $S E$ & $d f$ & $t$ & $p$ \\
\hline \multicolumn{6}{|l|}{ Fixed effects: } \\
\hline $\mathrm{X}$ & $0.0492[0.0215 ; 0.0769]$ & 0.0141 & 7.68 & 3.48 & .009 \\
\hline$x^{3}$ & $-0.0056[-0.0081 ;-0.0030]$ & 0.0013 & 5.37 & -4.32 & .006 \\
\hline Intercept & $0.0023[-0.0132 ; 0.0177]$ & 0.0079 & 4.84 & 0.29 & .787 \\
\hline
\end{tabular}


Table 9.

All fixed effects and related statistics from mixed model analysis for dissimilarity focus.

\begin{tabular}{llrrrrr}
\hline \multicolumn{1}{c}{} & \multicolumn{1}{c}{$S[95 \% \mathrm{CI}]$} & $S E$ & $d f$ & $t$ & $p$ \\
\hline Fixed effects: & & & & & \\
& $\mathrm{X}$ & $0.0105[-0.0143 ; 0.0353]$ & 0.0127 & 6.62 & 0.83 & .437 \\
& $\mathrm{x}^{3}$ & $-0.0045[-0.0068 ;-0.0021]$ & 0.0012 & 4.91 & -3.73 & .014 \\
& & & & & \\
& Intercept & $0.0097[-0.0059 ; 0.0253]$ & 0.0080 & 5.18 & 1.22 & .276 \\
\hline
\end{tabular}

The bootstrapping procedure with 3000 iterations showed that the AoA was larger under the similarity focus, $0.11 S D^{2}, S E=0.02$, Bias $<-0.001,95 \% \mathrm{CI}[0.07,0.15]$, than under the dissimilarity focus condition, $0.01 S D^{2}, S E=0.01$, Bias $=0.002,95 \%$ CI $[0,0.03]$, with percentile confidence intervals that did not overlap. These results agree with the main analyses as well as the expectation that a dissimilarity focus would reduce the strength of assimilation overall compared to a similarity focus. In agreement, the window of assimilation was also found to be wider in the similarity focus condition, 5.94SD, $S E=0.21$, Bias $=-0.01,95 \%$ CI $[5.48,6.31]$, than in the dissimilarity focus condition, 3.07SD, $S E=1.14$, Bias $=-0.29,95 \%$ CI $[0,4.24]$, with percentile confidence intervals that once again did not overlap. Taken together these results provide convincing evidence that a difference in similarity focus affects both the strength of the aggregated assimilative effects as well as shifting the window in which assimilation is the dominant tendency.

\section{Discussion}

This replication study produced results that were largely in line with the previous study, but this time the focal test did reveal a clear and significant effect in the expected direction. Separate analyses showed that the positive linear effect, a sign of assimilation, was only found in the similarity focus condition, but did not appear in the dissimilarity focus condition. Visual 
inspection of the resulting patterns also clearly showed more pronounced assimilative effect in the former than the latter condition. Lastly, the bootstrapping tests affirmed the previously found difference in the windows of assimilation, with a larger WoA in the similarity focus condition than the dissimilarity focus condition. In addition, it also showed a more convincing difference between the areas of assimilation as a whole.

The results thus show a clear shift in the comparative pattern when focusing on similarities versus differences, in line with the theoretical mechanism underlying the SAM and the pattern predicted in the introduction for a propensity altering moderator. More importantly, the results highlight the importance of standard selection for the investigation of moderating effects. There is no simple flip in the pattern from only assimilative effects to only contrastive effects, rather a shift in focus from similarities to dissimilarities corresponds to a shift in the window in which assimilation is likely to occur, while also reducing the aggregated strength of assimilation in this same window. However, even under a dissimilarity focus contrast effects do not dominate in comparisons with moderate standards in this context. In fact, the estimated windows of assimilation in these data indicate that one would expect to find consistent assimilation under a similarity focus, while simultaneously finding contrast under a dissimilarity focus, only for a small area between $1.5 S D$ and $3 S D$. Thus, only in this range would one expect to find a clear cross over effect when testing the moderator.

Shifting our focus to other potential moderators, the next study will show how other moderating variables can produce their own unique shifts in the dynamic interactive pattern of assimilation and contrast. In this case, the comparative pattern for the highly influential variable of shared or differing category information (Brewer \& Weber, 1994; Wänke et al., 2001; Mussweiler \& Bodenhausen, 2002; Brown et al., 1992) will be modelled. 


\section{Study 4}

Other moderating variables will often produce their own unique shifts in the dynamic interactive pattern of assimilation and contrast as they influence the equilibrium between these opposing effects in their own specific way. To illustrate this fact, the current study will turn to a different highly influential moderating variable, namely that of having a shared category. As with the manipulation of the comparative focus, the presence of shared or different category information has been hypothesized to influence the initial judgment of similarity or differences within the SAM framework (Mussweiler, 2003). Information about shared category membership will make it more likely that the two stimuli are judged as similar leading to assimilation, whereas differing category information will lead to dissimilarity testing and contrast (Mussweiler \& Bodenhausen, 2002). However, others have suggested that large differences between the target and the standard may cause cross-category comparisons be deemed as less diagnostic (Brown et al., 1992), potentially causing respondents to disregard the information altogether.

The current study will model the dynamic interactive pattern in response to same and cross-category comparisons to evaluate how this variable influences the entire dynamic interactive pattern for trustworthiness ${ }^{8}$. More specifically, it will model responses in the presence of standards of the same race compared to standards of a different ethnicity using the CJT-41 in a pre-registered study (https://aspredicted.org/xt4sq.pdf). We expect a positive linear effect and a negative cubic effect as reflective of assimilation to moderate and contrast from extreme standards. The category of the standard (same versus different ethnicity) should be found to significantly alter this pattern as seen in the improvement of the model fit when this condition

\footnotetext{
${ }^{8}$ Two studies also attempted to use the extraversion dimension, but failed to create multiple items that could sufficiently measure the basic dynamic pattern precisely enough even in the control condition. These studies are, of course, available on the OSF page.
} 
variable and all interactions are added. More specifically, if the differing category information increases the likelihood of contrast, we would expect the modified pattern to reflect this with a shift towards a narrower Window of Assimilation (WoA) and reduced Area of Assimilation (AoA). However, if the cross-category standards attenuate both comparative tendencies, we might see a similar WoA, if computable at all, but with a reduced AoA as described in the introduction for a strength altering moderator.

\section{Method}

Participants. In line with Study 3a, a sample of 957 self-identified white respondents completed the survey through the Prolific platform for a reward of $£ 1.50$ per respondent. This sample consisted of $54 \%$ females and ranged from 18 to 80 years of age $(M=35.96, S D=$ 13.79).

Category Manipulation. The CJT-41 was again implemented like in Study 2b, however this time participants were randomly assigned to either a same or different category condition. In the same category condition both the target and the standard would be White in all trials, whereas in the different category condition the target would be White, but the standards would be Black.

Stimuli. Eighty-one new faces were made in the same fashion as in Study 2a. The only exception is that the created IDs were duplicated and transformed to create standards that represented either White or Black faces around the category average. Targets on the other hand were the White faces in both category conditions.

Additional measures. Initially, the basic demographics of age, sex, and education were again measured after which the participants again described shapes they could see on an image. It is conceivable that some participants in the different category condition would be motivated to 
ignore the comparison standard all together to avoid the possibility of making unfavorable comparisons to a minority group and appearing prejudiced. For this reason, the Internal and External Motivation to Respond without Prejudice Scale (IMS $\alpha=.84$; EMS $\alpha=.79$; Plant \& Devine, 1998) was also included for exploratory analysis. The IMS includes 5 items like "I am personally motivated by my beliefs to be non-prejudiced toward Black people" while the EMS includes 5 items such as "I try to hide any negative thoughts about Black people in order to avoid negative reactions from others". Responses to both subscales are given on a Likert scale ranging from 1 (strongly disagree) to 9 (strongly agree). Scores are averaged and with higher scores indicating stronger motivations. For use in the analysis, these scores were further centered so that they ranged from -4 to 4 . Finally, participants were again asked to report what they believed the study had been about. In addition, the data-quality item and an ethnicity item was this time also included to make sure only white respondents were part of the final sample.

Procedure. Before taking part, participants were fully informed about the data storage policy, procedure, and their rights after which they gave their consent. The image identification question was administered first followed by the basic demographics. After this the participants were assigned to either the same or different category condition and received an explanation of the CJT including two practice trials. Following this, the main batch of 41 items were administered. After this main task, the suspicion item was administered followed by the ethnicity item. Finally, the data-quality item was answered and participants were thanked, debriefed and compensated.

Data treatment. Once again, non-numeric or empty responses were removed (2.4\%) along with all trials in which the judgment target was not correctly identified (1.7\%). Remaining scores were z-transformed per participant where possible and truncated above 3 or below -3 
(7.4\%). Responses to reverse coded items were then flipped. In total, these criteria led to the exclusion of $11.4 \%$ of trials. Furthermore, 29 participants indicated low self-reported data quality and 21 respondents indicate they did not identify as white despite the exclusion criteria used during recruitment. These respondent together with the other criteria led to the exclusion of 114 participants in total, leaving a final sample of 843 .

\section{Results}

Pre-registered analyses similar to those run for Study $3 a$ \& $3 \mathrm{~b}$ were performed by comparing two mixed models regressions using ML for model comparison. The two models again included linear and cubic fixed effects for the extremity steps with a similar structure of orthogonal uncorrelated random slopes and intercepts for participants and for items. One of these two models additionally included fixed effects for the condition variable and all interactions, allowing it to account for variation in the comparative outcomes due to the presence of shared or different category information. Results showed that the category condition indeed significantly influenced the comparison pattern, $\chi^{2}(3)=10.22, p=.017$. However, the descriptive curve, rather than showing stronger contrast for comparison with differing category memberships as was found for the comparative foci, only reflected a decrease in both assimilation and contrast (Figure 12).

Indeed, running separate models using REML for both conditions separately showed that only the shared category condition (Table 10) showed both a linear and cubic effect in the expected direction, while the different category condition did not show any signs of comparison whatsoever (Table 11). As the differing category membership showed no signs of comparison effects at all, no comparisons could be made regarding the Areas of Assimilation nor the Windows of Assimilation. 


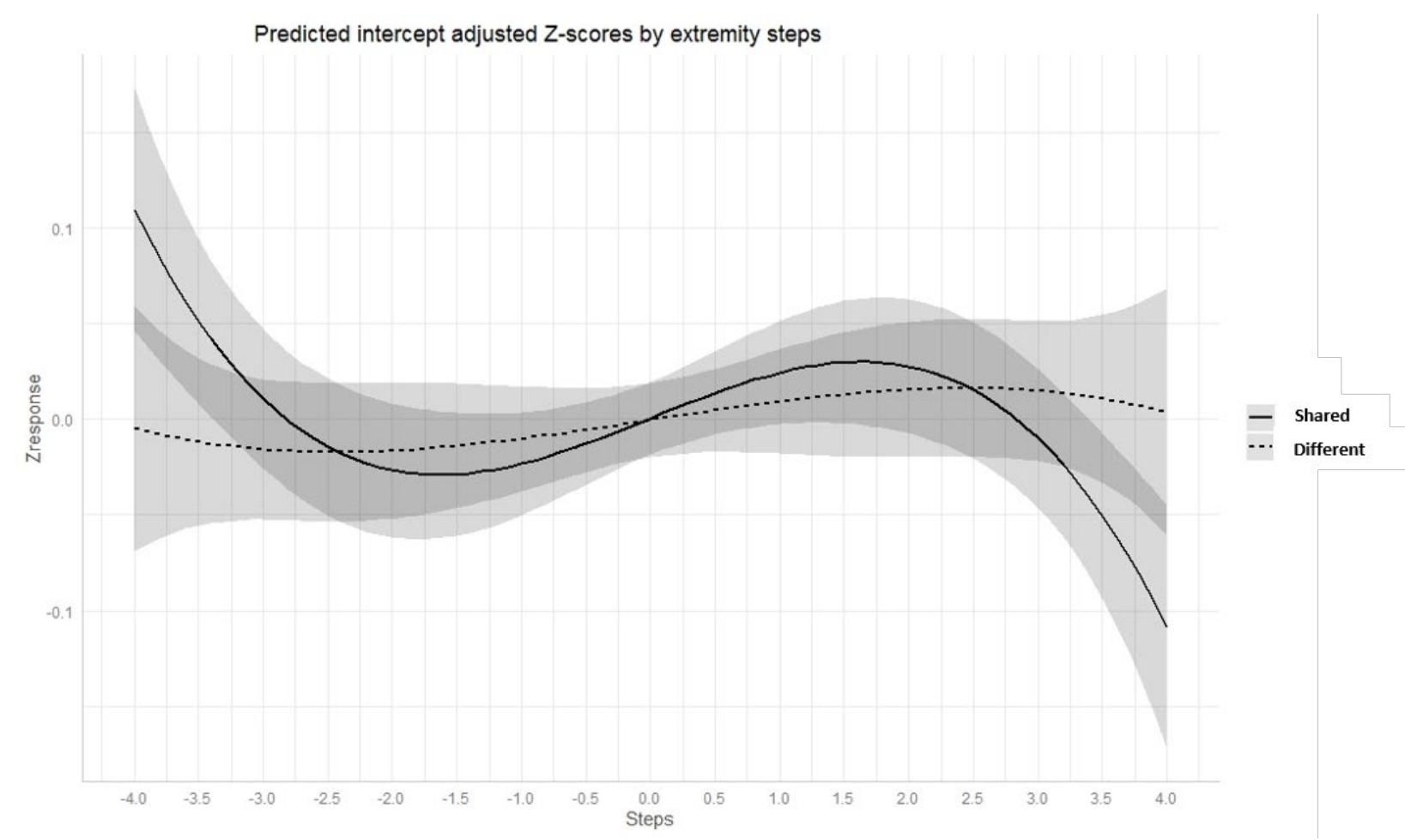

Figure 12. Predicted intercept adjusted marginal z-scores for the trustworthiness dimension separately for the different category conditions at each extremity step and their predicted $95 \% \mathrm{CI}$

As some respondents may be motivated to reduce their use of the standards in the different category condition in an attempt to hide potential prejudiced thoughts about the Black standards, the potential moderating role of one's internal and external motivation to respond without prejudice (Plant \& Devine, 1998) was investigated in an exploratory fashion. To this end, the centered IMS and EMS were added as moderators in separate mixed models analyses using REML for respondents in the different category condition. Random effects were identical to those used in the previous analyses. Although the external motivation did not affect the response patterns in any way, the internal motivation showed some evidence of a small moderating role within a purely linear model, $t(404.79)=-2.40, p=.017, B=-.005,95 \% \mathrm{CI}$ $[0.06,0.15]$. Follow up analyses showed that for the lowest quantile of respondents (scoring from -4 to -1.6 on the IMS), a purely assimilative effect was present, $t(12.89)=-2.47, \mathrm{p}=.028, \mathrm{~B}=$ 
$.0223,95 \%$ CI $[0.0046,0.0401]$. This effect was not there for the other quantiles, which also all significantly differed from the effect present in the lowest quantile, all $p \mathrm{~s}<.024$. This finding enhances concerns that the attenuation of the comparative effects may be the result of some respondent's unwillingness to use the comparative information from Black faces in an attempt to not appear prejudiced in any way. The safest way to do this is to ignore the comparative information as it is not directly relevant to the task. However, participants who were the least internally motivated by this factor still did not show the expected pattern, but rather showed a purely assimilative effect as opposed to the predictions of increased contrast.

Table 10.

All fixed effects and related statistics from mixed model analysis for the shared category condition.

\begin{tabular}{llrrrrr}
\hline & & $B[95 \% \mathrm{CI}]$ & $S E$ & $d f$ & $t$ & \\
\hline Fixed effects: & & & & & & \\
& $\mathrm{x}$ & $0.0271[0.0062 . ; 0.0479]$ & 0.0106 & 22.11 & 2.55 & .018 \\
& $\mathrm{x}^{3}$ & $-0.0034[-0.0049 ;-0.0019]$ & 0.0008 & 432.64 & -4.32 & $<.001$ \\
& & & & & .40 & .709 \\
\hline
\end{tabular}

Table 11.

All fixed effects and related statistics from mixed model analysis for the different category condition.

\begin{tabular}{|c|c|c|c|c|c|}
\hline & $B[95 \% \mathrm{CI}]$ & $S E$ & $d f$ & $t$ & $p$ \\
\hline \multicolumn{6}{|l|}{ Fixed effects: } \\
\hline $\mathrm{x}$ & $0.0108[-0.0231 ; 0.0447]$ & 0.0173 & 6.93 & 0.63 & .552 \\
\hline $\mathrm{x}^{3}$ & $-0.0007[-0.0039 ; 0.0025]$ & 0.0016 & 5.09 & -0.43 & .684 \\
\hline Intercept & $0.0033[-0.0139 ; 0.0204]$ & 0.0088 & 5.11 & 0.38 & .723 \\
\hline
\end{tabular}

\section{Discussion}

Although the expected S-shaped pattern of assimilation to moderate and contrast from extreme standards was again found in the same category condition, no such pattern emerged in the different category condition. Instead the current results speak to an attenuation of all types of 
comparative effects rather than showing increased signs of contrast that some of the literature would suggest (Brewer \& Weber, 1994; Wänke et al., 2001; Mussweiler \& Bodenhausen, 2002). Instead, the findings seem more in line with the notion that standards of a different category are deemed less relevant (Lockwood \& Kunda, 1997) and less diagnostic (Brown et al., 1992) causing respondents to disregard this information.

However, there were some additional signs that some participants may have been reluctant to use the comparative information due to the fact that it would entail making a potentially prejudiced judgment of an out-group member. Indeed, respondents with some internal motivation to appear non-prejudiced showed no comparative effects, while those lowest in this motivation did. However, rather than showing the expected pattern or increased contrast effects, they showed a purely assimilative pattern. This result, though not predicted, can be partially explained when considering the possibility that the cross category comparison broadened the representation of the category under consideration to include not just White individuals, but all people. Indeed, when included in a category, a-typical exemplars and heterogeneous category information has been suggested to lead to increased assimilation (Bless \& Schwarz, 2010) by easing the inclusion of a broader range of information. The reason such effects are not widely reported in the literature might be due to the specific categories used in the current work, namely Black and White faces. Using less socially sensitive categories may reduce motivational drives to not engage with the comparative information, though such boundary conditions have not yet been well defined. Although a sound possible explanation for the unexpected data pattern, these post-hoc explanations should be considered as such and with some caution as to not give them too much weight, as many other factors may have played a role. For instance, the trustworthiness dimension may be confounded with the ethnicity manipulation to some extent, as recent work 
has highlighting that this dimension may be processed differently across ethnic lines

(Charbonneau et al., 2020).

Regardless of the cause of the moderation, the change in the comparative pattern found here is a clear example of how different moderators can affect the comparative pattern in their own unique ways, while also emphasizing the need to model multiple extremity steps in order to reach accurate conclusions. Where Study $3 \mathrm{a}$ and $3 \mathrm{~b}$ emphasize the possibility of misidentifying increased assimilation as a null effect, the current study shows that true attenuation of all comparative effects would be difficult to distinguish from these former findings if only a narrow bands of standard extremities up to $1.5 S D$ would have been used. In this range, both the effects of the comparative focus and the category information variable have seemingly similar effect. Only when the full pattern is revealed do we clearly see how they are fundamentally different and one leads to increased contrast where the other reduces all tendencies.

\section{General discussion}

Research investigating the effects of external standards of comparisons on the formation of social judgments has often either ignored or arbitrarily dichotomized the relative standing of the standard in relation to the judgment target as simply either moderate or extreme. However, insufficiently accounting for the clearly continuous nature of the comparison standards extremity and its fundamental relationship to the expected outcome of the judgment will lead to inaccurate predictions and inferences. In addition, such approaches prevent strong tests of theory as posthoc judgments of the standards operationalization remain an ever present obstacle for falsification and the generalizability of results. The current work has offered a new framework for this type of comparative research which acknowledges that these influences of comparison standards on social judgments are inextricably linked to the exact standards, judgments, and 
context that evoke them. It has done so by modelling responses of different judgments in the presence of standards that vary across a wide range of values on the judgment dimension. The balance of the unique interactive patterns of assimilative (towards the standard) and contrastive forces (away from the standard) that dynamically influence the average response tendency at each point in the measured range of standard extremities (the extremity spectrum) varied widely depending on the exact item that was considered, but proved stable at the level of the judgment dimension. Finally, at this judgment dimension level, the modelling of this dynamic interactive pattern was shown to be able to offer stronger tests of theoretically interesting variables in future research.

Study 1 showed that the sensitivity of the comparative effects to the judgment being made, reported in previous work (Barker et al., 2020), was not caused by simple issues of standard calibration, but that these item level influences affect the propensity for assimilation or contrast across the entire spectrum of values for standard extremity that were tested. However, on the level of the judgment dimensions as a whole, modelled in Study 2, the theoretically expected aggregate pattern was present for all dimensions and of a similar shape, with standards extreme enough to elicit consistent aggregated contrast effects emerging around $3 S D$ from the target and assimilation proving to be strongest on average around $1 S D$ to $2 S D$. By moving away from the dichotomous terms of moderate or extreme, it becomes obvious that the relative standing of the standard influences the propensity for either assimilation on contrast to occur in every comparative judgment. Thus, assimilation and contrast should not be thought of in isolation, but as a complete dynamic interactive pattern in which both tendencies can occur to some degree at the level of a single judgment. Only as an average interplay of the two forces at a specific point in the spectrum of extremity values will consistent assimilation or contrast appear. 
Which will dominate at this aggregate level is fundamentally linked to the extremity of the standard, judgment, and context that gave rise to the comparison. Therefore, results from the existing literature, which often uses limited items and standards, should be thought of as representing only a sliver of the dynamic pattern for a specific judgment context, restricting inferences to exactly this snapshot of the process. Until such findings are revisited using a framework similar to the one presented here, they cannot be assumed to reflect the process across the values a standard can take. Future researchers would be advised to select a broad range of standard extremities and include this variable within their models to make the most accurate inferences, but should at minimum report the relative standing of their standards so results can be meaningfully compared across studies.

A similar issue surrounds the limited use of items in these investigations, as became clear in the initial studies based on the variation seen in Barker et al. (2020). Although the substantial differences between the items in the current work makes it impossible to provide strong claims about the conditions that caused these fluctuations, past research has emphasized the highly sensitive nature of similarity judgments to linguistic context and conversational norms (Roth \& Shoben, 1983; Barsalou, 1982). For instance, the inclusion of a single noun can emphasize one aspect of similarity over other potential dimensions (Landau et al., 1988). Hence, the strong variation in linguistic context across the items could have emphasize different aspects of how the stimuli might be similar or dissimilar. For instance, the single dominance and trustworthiness items in study $1 \mathrm{~b}$ and $\mathrm{c}$ strongly reference the ways in which the target may relate to their social surroundings by "deceiving" or "enforcing", while the item in study 1a for extraversion merely involves the targets own behavior. Highlighting different social relations can indeed have profound effects on whether assimilation or contrast occurs even when the same stimuli are 
involved (Schwarz, \& Bless, 1992a). Past research has, for instance, shown how emphasizing a politician's impartiality can cause contrast effects, while his previous party affiliation can lead to assimilation (Bless, \& Schwarz, 1998). Hence, while “enforcing one's opinion" may highlight the target's separation from other individuals in their opinions leading to increased contrast, the term "deceiving others" may emphasize how the two can be influenced to be of a similar mind, showing their mutability which has been suggested to increase assimilation (Schwarz \& Bless, 2007).

Whatever the exact cause, it is clear from the profound variation seen across studies that researchers must aim to include multiple items and show caution when interpreting findings if only few were included. Even within the current work, which sampled multiple items, the uncovered comparative patterns strictly only extend to the population of dimensions, items, stimuli, samples, and the contexts in which they were measured. Hence the specific patterns uncovered here are likely a complex combination of all these factors and the resulting task demands. They cannot be generalized without making strict assumptions about their relation to other judgment contexts, which is unlikely considering the strong context sensitivity of similarity judgments (Medin et al., 1993). More so, one should consider that the single items and dimensions used in the studies were purposely selected from previous work exactly because they showed consistent comparison effects, where other items appeared to elicit none (e.g. Likability; Barker et al., 2020). Whether the comparative patterns and estimated turning points presented here would be comparable for other facial dimensions or even extend beyond the facial domain itself will need to be the subject of future research, although the relative consistency of results found here at the dimensional level seems encouraging. 
The culmination of all these points can clearly be seen in the results found in the final section, showing how this new holistic framework can offer stronger tests of other theoretically important variables providing more accurate inferences. Not only did Study $3 \mathrm{a}$ and $3 \mathrm{~b}$ reaffirm the robustness of the similarity focus as a moderator using explicit manipulations, they also clearly revealed that, rather than leading to only assimilation or only contrast, such variables cause a shift in the dynamic interactive pattern affecting the equilibrium at each point in the extremity spectrum. This leads to the suppression of assimilative tendencies and narrowing of the window of assimilation under a dissimilarity focus compared to the similarity focus. However, even under a dissimilarity focus, assimilative effects do still occur for more moderate standards, albeit with significantly reduced aggregated strength. As a result of this shift, wide stretches of the extremity spectrum seemingly do not produce consistent contrast under a dissimilarity focus (0SD to $1.5 \mathrm{SD}$ ), nor assimilation under a similarity focus (3SD or above). In fact, only narrow bands of standards from this spectrum would be expected to find the classical pattern, i.e. assimilation for a similarity and contrast for a dissimilarity focus, only in the stretch of roughly 1.5SD to $3 \mathrm{SD}$ from the target. Again, this non-linearity is expected if a moderating variable influences the likelihood of one comparative outcome over another rather than strictly excluding one of these tendencies altogether, which is unlikely to occur for variables that cannot be directly and completely influenced due to the imperfect construct validity of any such manipulation (Cronbach \& Meehl, 1955; Campbell, 1957; Cook \& Campbell, 1979).

If one only made claims regarding the difference between the two foci, i.e. outcomes for a similarity versus dissimilarity focus for the same standard, this would not be as problematic, although one may note that the size of the relative difference need not be equal at all steps. However, when referring to a single focus in isolation or in comparison to a control condition, 
one may detect no noticeable comparative effects if the standards used are representative of the point in the extremity spectrum where assimilation and contrast effects cancel each other out. This can lead to erroneous conclusions that one condition does not cause assimilation nor contrast, when in a sense it is causing equal amounts of both. Although these findings do not speak directly to the efficacy of the more common comparative priming procedure that was the focus in the recent meta-analysis by Gerber et al. (2018), they do suggest that situations where contrast is found for a dissimilarity focus and no assimilation is found under a similarity focus should not be surprising. In fact, it may even be expected if the individual studies create a context in which contrast is more dominant overall, as was indeed the case in their analysis.

However, distinguishing these potential phantom comparison effects from true null effects is impossible if only a narrow band of extremity steps are considered in an analysis, as became apparent in Study 4 investigating cross-category comparisons, or the lack there off. Rather than producing the expected increase in contrast effects, the presence of standards from a differing category resulted in a true attenuation of both comparative effects across the extremity spectrum. Although the differences between the effects of the comparative foci and this attenuation are clear when viewed across the dynamic pattern, single point measurements could easily result in identical patterns. For instance, one may notice that when focused just on standards up to about $1.5 S D$, the difference between the two conditions is largely similar to the difference found between the two comparative focus conditions in Study 3a and 3b. This illustrates that conditions under which assimilation and contrast are present in equal measure, phantom comparisons, can seem similar to those in which comparative information is not used at all, and with it the benefits of a more holistic framework. However, the CJT paradigm is but one approach that can be taken to investigate comparison patterns in this holistic fashion. Any 
paradigm which includes multiple comparison standards, with a relatively precise manipulation of their extremity information, should be capable of achieving similarly strong tests. In addition, many investigations may not need the same granularity that the CJT provides, and could further reduce strain on participants by only including certain bands of extremity in which one expects the effect to occur based on previous work. Future investigations should take this to heart and design their investigations with this complex dynamic in mind, even if standard extremity is not the primary focus of the investigation or run the risk of misinterpreting results.

We should note here that the attenuation of all comparative effect found in Study 4 for the cross-category comparisons itself, although an illustrative pattern, is not predicted by all theoretical accounts (Brewer \& Weber, 1994; Wänke et al., 2001; Mussweiler \& Bodenhausen, 2002), and may have a number of causes. For instance, since the current paradigm does not explicitly require participants to compare the stimuli, similarity may also function as a standard selection mechanism with cross-category comparisons being disregarded more often. This could be due to them being deemed no longer relevant or informative (Lockwood \& Kunda, 1997; Brown et al., 1992) or because some participants may be motivationally driven to ignore the potentially socially sensitive consequences of interracial comparisons. Furthermore, the evaluative dimension of trustworthiness may be too closely related to the ethnic categories used in the current work, with recent research highlighting that the visual cues of facial trustworthiness may be processed differently across ethnic lines (Charbonneau et al., 2020). Therefore, the exact reason for the attenuation found in the current data remains unclear, and the findings should not be generalized to hastily. It is possible that when cross category comparisons do occur, or when other judgment dimensions are used, they do increase contrast.

Notwithstanding, these potential issues offer interesting avenues for future research that may 
ultimately uncover new insights into the comparative process. One interesting question is whether the attenuation in the current case, whatever the cause, occurs previous to any comparison taking place at all as described above, or if it is the product of later alterations made by corrective processes, as has been suggested to be the case for some non-informative comparisons (Gilbert et al., 1995).

Another observation that may be of theoretical importance is that the baseline pattern for Trustworthiness in Study $2 \mathrm{~b}$ and the shared category condition in Study 4, which do not include any instructions regarding comparative foci, show a dynamic pattern and Window of Assimilation most similar to the similarity focus in Studies 3a \& 3b. This may suggest that within the current context of the CJT the default focus of comparison might be one of similarities as suggested by Mussweiler (2003). However, this is in opposition to the tendency towards contrast effects suggested by Gerber et al. (2018), which found these to be dominant across studies. This discrepancy could be a result of specific task demands in the CJT-41 paradigm. For instance, feelings of trust or distrust have been found to affect the comparative process (Posten \& Mussweiler, 2013). Similarly, the simultaneous presentation of standards, rather than sequential presentation, may itself induce more assimilation than contrast (Wedell et al., 1987). These sensitivities to methodological variations restrict the conclusions that can be made based on the current findings concerning the default or dominant comparative outcome. Nevertheless, it should equally caution us from making such claims based on the aggregated strength of effects across existing studies. The dominance of contrast across the literature reported in Gerber et al. (2018) may also simply be another indication that the standards and contexts used in these studies are those which are more likely to induce contrast effects. Hence, aggregated effects of any kind will not give a definitive answer regarding which tendency is more dominant, all else 
being equal. Instead, additional dedicated lines of research into the detailed underlying process that give rise to these effects would be necessary.

\section{Limitations}

In addition to the limitation already discussed, the current paradigm suffers from the especially small effect sizes it produces. This may partially explain why previous research has occasionally struggled to find consistent effects, which in turn could simply be the inevitable result of the dynamic process itself in which opposing effects cancel each other out to some degree at any point. However, it could also be a result of the minimal information the facial stimuli offer. Although they offer precise control to the researcher, they leaves little for participants to base their judgments on. Indeed, prominent models like the SAM (Mussweiler, 2003) have emphasized the role of the activation of standard-consistent knowledge and resulting confirmatory processing of the target. Arguably, the limited knowledge and information available from faces might result in minimal comparison effects. The paradigm, thus, also represents a minimal scenario of comparative judgments which might underestimate the size of comparison effects as they happen in other contexts. Indeed, the bulk of comparison research has focused on self-related judgments where one has an incomparable and intimate knowledge of the target (the self) while also presenting well known individuals or detailed descriptions of standards (Mussweiler, et al., 2004a), providing ample additional information that one can draw from $^{9}$. Expanded versions of such paradigms may produce stronger assimilative and contrastive effects, which could also result in different levels of extremity being optimal for producing the respective outcomes. Future research investigating domains other than facial judgments as well

\footnotetext{
${ }^{9}$ Problematically, this information is likely to additionally contain many other theoretically relevant moderating variables, which can only be accounted for if a wide representative sample of similar standards is included.
} 
focusing on self-related judgments will be needed to test the universality of the uncovered patterns and provide guidelines in other comparative contexts. For instance, including additional knowledge of the target in some unobtrusive may increase the activation of standard-consistent knowledge and the strength of the comparative effects. With this alteration, the increased strength of both comparative effects would be expected to increase the Area of Assimilation specifically, while the Window of Assimilation should remain unchanged as the initial holistic assessment should not be directly affected.

Another issue with the current operationalization of the facial dimensions is that they do not remain fully orthogonal to all other facial dimensions. Hence, despite the fact that the control exerted on the dimension of interest remains highly precise compared to other methods, secondary dimensions may also have been inadvertently manipulated to a lesser degree. This is indeed an inherent issue with the use of facial dimensions, as noted by Oosterhof and Todorov (2008) themselves. This could potentially increase the perceived dissimilarity between the more extreme standards and the target on more than just the focal dimension. To some degree this will be true of any manipulation of the underlying dimensions as, again, any instrumental variable will not have perfect construct validity (Cronbach \& Meehl, 1955; Campbell, 1957; Cook \& Campbell, 1979). Although this is likely less of an issue for the precise and structured creation of comparison standard used here compared to most previous investigations that use hand-picked stimuli (e.g. Shirley Temple vs. Hitler in Herr, 1986), this point should not be overlooked when interpreting the results and may have altered the patterns to show narrower windows of assimilation.

Similarly, the patterns presented here are limited by the populations in which they were found and may not reflect a universal tendencies. Although both online and lab samples were 
collected from different national populations ([masked], the US and the UK), all were from western backgrounds, a common issue in psychological research (Henrich, Heine \& Norenzayan, 2010). It is quite possible that non-western samples may produce remarkably different patterns. For instance, a more collectivist cultural upbringing has been suggested to increase the general tendency to compare especially with upward standards (Chung \& Mallery, 1999; Baldwin \& Mussweiler, 2018) and the related interdependent self-construal can result in less contrastive tendencies (Cheng \& Lam, 2007). Future research should be encouraged to include non-Western populations in order to identify the universality of these comparative patterns.

Finally, although the current work clarifies many theoretical ambiguities, it does not clearly favor models that predict separate assimilative or contrastive judgments (e.g. the SAM; Mussweiler, 2003) from those that predict internal aggregation of the dual forces (e.g. the IEM; Schwarz \& Bless 2007), since both could predict the proposed S-shaped dynamic pattern depending on their exact parameters. However, similar paradigms may in the future be able to better disentangle these possibilities by focusing on the distinct pattern of response variation that would be predicted at different points in the extremity spectrum. Similarly, the paradigm can be adjusted to manipulate the informational content of the standard and target separately, which may be a second avenue to distinguishing the predictive strength of the many different comparison models. Thus, although the current work itself does not give a definitive answer about which model is more suited to describe the comparative process, it does offer a valuable and flexible tool for future work to resolve these questions.

\section{Beyond binaries}

Notwithstanding the limits of the findings, the current work is a first step towards producing clearer boundaries for moderating variables and is the first to provide a detailed 
description of the underlying dynamic pattern of assimilation and contrast in any context. Although the current work has only focused on comparative outcomes, other branches of comparative research might similarly benefit from adopting a more holistic approach. For instance, the extremity of standards has recently been suggested as being of influence in the comparative effects on self-esteem (Fardouly et al., 2017) and motivation (Diel \& Hoffmann, 2019). In light of these advances, a broader selection of standards will need to be consider in these areas as well, with explicit modelling of the relative standing if non-linear dynamics similar to the ones presented here are present. Even in absence of such complex dynamics, many, if not all comparison areas should report the relative standing of their standards to aid comparison between studies, generalization and replication efforts.

In addition, other abstractly defined and arbitrarily dichotomized variables in the comparison literature (e.g., Warm vs. Cold, Steinmetz \& Mussweiler, 2011; Certain vs. Uncertain, Pelham \& Wachsmuth, 1995; Fluent vs. Disfluent, Häfner \& Schubert, 2008; or Minority vs. Majority influence, Brewer \& Weber, 1994) may benefit from being treated as continuous. Indeed, these variables share similar issues regarding the vagueness of their boundary conditions, again leaving post-hoc judgments of the aptness of their operationalization as a safeguard against falsification. A similar approach as outlined in this work would side-step these concerns, provide clearer guidelines, and easily distinguish any phantom comparisons at the conceptual boundary between these variables from true null effects. This is not to say that every investigation must sample the entire range of the moderator per se, or even fully move away from simplifying terms like moderate or extreme. Rather, it means that, at minimum, they must avoid including only very narrow bands of the moderating variable. For instance, one might choose to define 'moderate' as between $1 S D$ and $2 S D$ and sample standards equally within this 
range. This will not only improve generalizability beyond the exact standard extremity used, but will simultaneously bindingly define the boundaries within which researchers expect a certain outcome making findings more easily verifiable by others.

These issues are, of course, not unique to comparative research. Indeed, a general move away from binary thinking towards continuous manipulations will likely also strengthen inferences, make boundary conditions explicit, and uncover non-linear relationships in other fields. For instance, although the current work mainly focusses on social comparative judgments displayed simultaneously, similar non-linear effects have been suggested to take place sequentially in comparative settings (Barker \& Imhoff, 2021) as well as in the social perception literature where only a single stimuli is presented at a time (Cogan et al., 2013). Although the current work may not speak directly to these sequential context effects, a similar holistic and continuous approach could help better define their role and create more accurate models of social perception in general.

\section{Conclusion}

In conclusion, the current work extends the theoretical prediction of assimilation to moderate and contrast from extreme standards to the common everyday life judgments of facial characteristics, while simultaneously uncovering the previously obscured dynamic interactive pattern of assimilation and contrast. This finding, along with its demonstrated sensitivity to changes in study design, such as item selection and comparative mind-set, demonstrates the limitations of previous designs with limited item and standard selection. Although this high sensitivity also means that the precise patterns found here are likely to be substantially different in different research contexts, this fact only emphasizes the points raised regarding the limits of the generalizability of previous findings, and strengthens the call for a new standard of 
comparative research. As a whole, the current work has made a clear case for the more holistic framework described here, while also providing a basic paradigm that can be used for strong tests of theory by modelling the complete dynamic interactive patterns that underlie comparative outcomes.

\section{Open Practices}

The pre-registration documentation for the following studies are available:

Study 2a at: https://aspredicted.org/9h8tj.pdf

Study 2 b at: https://aspredicted.org/zc3at.pdf

Study 3a \& b at: https://aspredicted.org/tk46j.pdf

Study 4 at: $\underline{\text { https://aspredicted.org/xt4sq.pdf }}$

All data files, analyses and additional materials can be found at:

https://osf.io/sxq2c 


\section{References}

Ahrens, A. H. (1991). Dysphoria and social comparison: Combining information regarding others' performance. Journal of Social and Clinical Psychology, 10, 190-205. https://doi.org/10.1521/jscp.1991.10.2.190

American Psychological Association. (2017). Ethical principles of psychologists and code of conduct (2002, amended effective June 1, 2010, and January 1, 2017). https://www.apa.org/ethics/code/

Baldwin, M., \& Mussweiler, T. (2018). The culture of social comparison. Proceedings of the National Academy of Sciences, 115, E9067-E9074. https://doi.org/10.1073/pnas.1721555115

Ballew, C.C., \& Todorov, A. (2007). Predicting political elections from rapid and unreflective face judgments. Proceedings of the National Academy of Sciences. 104, 17948-17953. https://doi.org/10.1073/pnas.0705435104

Barker, P., Dotsch, R., \& Imhoff, R. (2020). Assimilation and Contrast in Spontaneous Comparisons: Heterogeneous Effects of Standard Extremity in Facial Evaluations. International Review of Social Psychology, 33, 11. http://doi.org/10.5334/irsp.402.

Barker, P., \& Imhoff, R. (2021) Connecting the Dots: Non-linear Patterns in the Presence of Symbolic and Non-symbolic Numerical Standards. Manuscript submitted for publication.

Barsalou, L. W. (1982). Context-independent and context-dependent information in concepts. Memory \& cognition, 10(1), 82-93. https://doi.org/10.3758/BF03197629

Bates, D., Maechler, M., Bolker, B., \& Walker, S. (2015). Fitting Linear Mixed-Effects Models Using lme4. Journal of Statistical Software, 67, 1-48. https://doi.org/10.18637/jss.v067.i01 
Berger S., Graham, N., \& Zeileis, A. (2017). Various versatile variances: An object-oriented implementation of clustered covariances in R, Working Papers, Faculty of Economics and Statistics, University of Innsbruck, https://EconPapers.repec.org/RePEc:inn:wpaper:2017-12.

Bless, H., \& Schwarz, N. (1998). Context effects in political judgement: Assimilation and contrast as a function of categorization processes. European Journal of Social Psychology, 28, 159-172. https://doi.org/10.1002/(SICI)10990992(199803/04)28:2<159::AID-EJSP860>3.0.CO;2-4

Bless, H., \& Schwarz, N. (2010). Mental construal and the emergence of assimilation and contrast effects: The inclusion/exclusion model. In Advances in experimental social psychology (Vol. 42, pp. 319-373). Academic Press. https://doi.org/10.1016/S00652601(10)42006-7

Brewer, M. B., \& Weber, J. G. (1994). Self-evaluation effects of interpersonal versus intergroup social comparison. Journal of Personality and Social Psychology, 66, 268. https://doi.org/10.1037/0022-3514.66.2.268

Brown, J. D., Novick, N. J., Lord, K. A., \& Richards, J. M. (1992). When Gulliver travels: social context, psychological closeness, and self-appraisals. Journal of Personality and Social Psychology, 62, 717. https://doi.org/10.1037/0022-3514.62.5.717

Buunk, A. P., Groothof, H. A. K., \& Siero, F. W. (2007). Social comparison and satisfaction with one's social life. Journal of Social and Personal Relationships, 24, 197-205. https://doi.org/10.1177/0265407507075410

Campbell, D. T. (1957). Factors relevant to the validity of experiments in social settings. Psychological Bulletin, 54, 297-312. http://dx.doi.org/10 .1037/h0040950 
Canty, A., \& Ripley, B. (2019). boot: Bootstrap R (S-Plus) Functions. R package version 1.3-24.

Cash, T. F., Cash, D. W., \& Butters, J. W. (1983). "Mirror, mirror, on the wall...?": Contrast effects and self-evaluations of physical attractiveness. Personality and Social Psychology, 9, 351-358. https://doi.org/10.1177/0146167283093004

Charbonneau, I., Robinson, K., Blais, C., \& Fiset, D. (2020). Implicit race attitudes modulate visual information extraction for trustworthiness judgments. Plos one, 15, e0239305. https://doi.org/10.1371/journal.pone.0239305

Cheng, R. W. Y., \& Lam, S. F. (2007). Self-construal and social comparison effects. British Journal of Educational Psychology, 77, 197-211. https://doi.org/10.1348/000709905X72795

Chung, T., \& Mallery, P. (1999). Social comparison, individualism-collectivism, and self-esteem in China and the United States. Current Psychology, 18, 340-352. https://doi.org/10.1007\%2Fs12144-999-1008-0

Cogan, E., Parker, S., \& Zellner, D. A. (2013). Beauty beyond compare: Effects of context extremity and categorization on hedonic contrast. Journal of Experimental Psychology: Human Perception and Performance, 39, 16-22. https://doi.org/10.1037/a0031020

Cook, T. D., \& Campbell, D. T. (1979). Quasi-experimentation: Design \& analysis issues for field settings. Boston, MA: Houghton Mifflin.

Corcoran, K., Epstude, K., Damisch, L., \& Mussweiler, T. (2011). Fast similarities: efficiency advantages of similarity-focused comparisons. Journal of Experimental Psychology: Learning, Memory, and Cognition, 37, 1280. https://doi.org/10.1037/a0023922 
Corcoran, K., Hundhammer, T., \& Mussweiler, T. (2009). A tool for thought! When comparative thinking reduces stereotyping effects. Journal of Experimental Social Psychology, 45, 1008-1011. https://doi.org/10.1016/j.jesp.2009.04.015

Corneille, O., Yzerbyt, V. Y., Pleyers, G., \& Mussweiler, T. (2009). Beyond awareness and resources: Evaluative conditioning may be sensitive to processing goals. Journal of Experimental Social Psychology, 45, 279-282. https://doi.org/10.1016/j.jesp.2008.08.020

Cronbach, L. J., \& Meehl, P. E. (1955). Construct validity in psychological tests. Psychological Bulletin, 52, 281-302. https://doi.org/10.1037/h0040957

Diel, K., \& Hofmann, W. (2019). Inspired to perspire: The interplay of social comparison direction and standard extremity in the context of challenging exercising goals. Social Cognition, 37, 247-265. https://doi.org/10.1521/soco.2019.37.3.247

Dunning, D., \& Hayes, A. F. (1996). Evidence for egocentric comparison in social judgment. Journal of Personality and Social Psychology, 71, 213. https://doi.org/10.1037/00223514.71 .2 .213

Dunning, D., Meyerowitz, J. A., \& Holzberg, A. D. (1989). Ambiguity and self-evaluation: The role of idiosyncratic trait definitions in self-serving assessments of ability. Journal of personality and social psychology, 57, 1082. https://doi.org/10.1037/00223514.57.6.1082

Epstude, K., \& Mussweiler, T. (2009). What you feel is how you compare: How comparisons influence the social induction of affect. Emotion, 9, 1. https://doi.org/10.1037/a0014148

Fardouly, J., Pinkus, R. T., \& Vartanian, L. R. (2017). The impact of appearance comparisons made through social media, traditional media, and in person in women's everyday lives. Body image, 20, 31-39. https://doi.org/10.1016/j.bodyim.2016.11.002 
Festinger, L. (1954). A theory of social comparison processes. Human Relations, 7, 117-140. https://doi.org/10.1177/001872675400700202

Gentner, D., \& Markman, A. B. (1994). Structural alignment in comparison: No difference without similarity. Psychological science, 5, 152-158. https://doi.org/10.1111/j.14679280.1994.tb00652.x

Gerber, J. P., Wheeler, L., \& Suls, J. (2018). A social comparison theory meta-analysis 60+ years on. Psychological Bulletin, 144, 177. https://doi.org/10.1037/bul0000127

Gibbons, F. X., \& Buunk, B. P. (1999). Individual differences in social comparison: Development of a scale of social comparison orientation. Journal of Personality and Social Psychology, 76, 129. https://doi.org/10.1037/0022-3514.76.1.129

Häfner, M., \& Schubert, T. W. (2009). Feel the difference! The influence of ease experiences on the direction of social comparisons. Journal of Experimental Social Psychology, 45, 291294. https://doi.org/10.1016/j.jesp.2008.09.008

Henrich, J., Heine, S. J., \& Norenzayan, A. (2010). Most people are not WEIRD. Nature, 466, 29-29. https://doi.org/10.1038/466029a

Herr, P. M. (1986). Consequences of priming: Judgment and behavior. Journal of Personality and Social Psychology, 51, 1106. https://doi.org/10.1037/0022-3514.51.6.1106

Herr, P. M., Sherman, S. J., \& Fazio, R. H. (1983). On the consequences of priming: Assimilation and contrast effects. Journal of Experimental Social Psychology, 19, 323340. https://doi.org/10.1016/0022-1031(83)90026-4

Imhoff, R., Woelki, J., Hanke, S., \& Dotsch, R. (2013). Warmth and competence in your face! Visual encoding of stereotype content. Frontiers in Psychology, 4 (386). https://doi.org/10.3389/fpsyg.2013.00386 
Kahneman, D., \& Miller, D. T. (1986). Norm theory: Comparing reality to its alternatives. Psychological Review, 93, 136. https://doi.org/10.1037/0033-295X.93.2.136

Kondo, A., Takahashi, K., \& Watanabe, K. (2013). Influence of gender membership on sequential decisions of face attractiveness. Attention, Perception, \& Psychophysics, 75, 1347-1352. https://doi.org/10.3758/s13414-013-0533-y

Kuznetsova, A., Brockhoff, P. B., \& Christensen, R. H. B. (2017). lmerTest package: Tests in linear mixed effects models. Journal of Statistical Software, 82(13), 1-26. https://doi.org/10.18637/jss.v082.i13

Landau, B., Smith, L. B., \& Jones, S. S. (1988). The importance of shape in early lexical learning. Cognitive development, 3, 299-321. https://doi.org/10.1016/08852014(88)90014-7

Lockwood, P., \& Kunda, Z. (1997). Superstars and me: Predicting the impact of role models on the self. Journal of personality and social psychology, 73, 91. https://doi.org/10.1037/0022-3514.73.1.91

Lüdecke, D. (2018). ggeffects: Tidy data frames of marginal effects from regression models. Journal of Open Source Software, 3, 772. http://doi.org/10.21105/joss.00772.

Lüdecke, D., Ben-Shachar, M., \& Makowski, D. (2020). “Describe and understand your model's parameters.” CRAN. https://doi.org/10.5281/zenodo.3731932

Markman, A. B., \& Gentner, D. (1993). Structural alignment during similarity comparisons. Cognitive psychology, 25, 431-467. https://doi.org/10.1006/cogp.1993.1011

Martin, R., Suls, J., \& Wheeler, L. (2002). Ability evaluation by proxy: Role of maximal performance and related attributes in social comparison. Journal of Personality and Social Psychology, 82, 781-791. https://doi.org/10.1037/0022-3514.82.5.781 
McFarland, C., Buehler, R., \& MacKay, L. (2001). Affective responses to social comparisons with extremely close others. Social Cognition, 19, 547-586. https://doi.org/10.1521/soco.19.5.547.19911

Medin, D. L., Goldstone, R. L., \& Gentner, D. (1993). Respects for similarity. Psychological Review, 100, 254-278. https://doi.org/10.1037/0033-295X.100.2.254

Meehl, P. E. (1990). Appraising and amending theories: The strategy of Lakatosian defense and two principles that warrant it. Psychological Inquiry, 1, 108-141. https://www.jstor.org/stable/1448768

Mendes, W. B., Blascovich, J., Major, B., \& Seery, M. (2001). Challenge and threat responses during downward and upward social comparisons. European Journal of Social Psychology, 31, 477-497. https://doi.org/10.1002/ejsp.80

Mussweiler, T. (2001). 'Seek and ye shall find': Antecedents of assimilation and contrast in social comparison. European Journal of Social Psychology, 31, 499-509. https://doi.org/10.1002/ejsp.75

Mussweiler, T. (2003). Comparison processes in social judgment: mechanisms and consequences. Psychological Review, 110, 472. https://doi.org/10.1037/0033295X.110.3.472

Mussweiler, T., \& Bodenhausen, G. V. (2002). I know you are, but what am I? Self-evaluative consequences of judging in-group and out-group members. Journal of Personality and Social Psychology, 82, 19. https://doi.org/10.1037/0022-3514.82.1.19

Mussweiler, T., \& Epstude, K. (2009). Relatively fast! Efficiency advantages of comparative thinking. Journal of Experimental Psychology: General, 138, 1. https://doi.org/10.1037/a0014374 
Mussweiler, T., \& Strack, F. (1999). Hypothesis-consistent testing and semantic priming in the anchoring paradigm: A selective accessibility model. Journal of Experimental Social Psychology, 35, 136-164. https://doi.org/10.1006/jesp.1998.1364

Mussweiler, T., \& Strack, F. (2000). The 'relative self': Informational and judgmental consequences of comparative self-evaluation. Journal of Personality and Social Psychology, 79, 23. https://doi.org/10.1037//0022-3514.79.1.23

Mussweiler, T., Rüter, K., \& Epstude, K. (2004a). The man who wasn't there: Subliminal social comparison standards influence self-evaluation. Journal of Experimental Social Psychology, 40, 689-696. https://doi.org/10.1016/j.jesp.2004.01.004

Mussweiler, T., Rüter, K., \& Epstude, K. (2004b). The ups and downs of social comparison: Mechanisms of assimilation and contrast. Journal of Personality and Social Psychology, 87, 832. https://doi.org/10.1037/0022-3514.87.6.832

Oosterhof, N. N., \& Todorov, A. (2008). The functional basis of face evaluation. Proceedings of the National Academy of Sciences, 105, 11087-11092. https://doi.org/10.1073/pnas.0805664105

Peer, E., Brandimarte, L., Samat, S., \& Acquisti, A. (2017). Beyond the Turk: Alternative platforms for crowdsourcing behavioral research. Journal of Experimental Social Psychology, 70, 153-163. https://doi.org/10.1016/j.jesp.2017.01.006

Pelham, B. W., \& Wachsmuth, J. O. (1995). The waxing and waning of the social self: Assimilation and contrast in social comparison. Journal of personality and social psychology, 69, 825. https://doi.org/10.1037/0022-3514.69.5.825 
Philippot, P., Schwarz, N., Carrera, P., De Vries, N., \& Van Yperen, N. W. (1991). Differential effects of priming at the encoding and judgment stage. European Journal of Social Psychology, 21, 293-302. https://doi.org/10.1002/ejsp.2420210403

Posten, A. C., \& Mussweiler, T. (2013). When distrust frees your mind: The stereotype-reducing effects of distrust. Journal of Personality and Social Psychology, 105, 567. https://doi.org/10.1037/a0033170

R Core Team (2018). R: A language and environment for statistical computing [Computer software]. R Foundation for Statistical Computing, Vienna, Austria. Retrieved from https://www.R-project.org/.

Raat, A.N., Kuks, Jan B.M., Van Hell, E.A., \& Cohen-Schotanus, J. (2013). Peer influence on students' estimates of performance: Social comparison in clinical rotations. Medical Education, 47, 190-197. https://doi.org/10.1111/medu.12066

Roth, E. M., \& Shoben, E. J. (1983). The effect of context on the structure of categories. Cognitive psychology, 15, 346-378. https://doi.org/10.1016/0010-0285(83)90012-9

Schwarz, N., \& Bless, H. (1992b). Constructing reality and its alternatives: Assimilation and contrast effects in social judgment. In L. L. Martin \& A. Tesser (Eds.), The construction of social judgments (pp. 217-245). Hillsdale, NJ: Lawrence Erlbaum Associates, Inc.

Schwarz, N., \& Bless, H. (1992a). Scandals and the public's trust in politicians: Assimilation and contrast effects. Personality and Social Psychology Bulletin, 18, 574-579. https://doi.org/10.1177/0146167292185007

Schwarz, N., \& Bless, H. (2007). Mental construal processes: The inclusion/exclusion model. In D. Stapel \& J. Suls (Eds.), Assimilation and contrast in social psychology (pp. 119-141). Philadelphia, PA: Psychology Press. 
Simonsohn, U. (2018). Two lines: A valid alternative to the invalid testing of $\mathrm{u}$-shaped relationships with quadratic regressions. Advances in Methods and Practices in Psychological Science, 1, 538-555. https://doi.org/10.1177/2515245918805755

Smith, W. P., \& Sachs, P. R. (1997). Social comparison and task prediction: Ability similarity and the use of a proxy. British Journal of Social Psychology, 36, 587-602. https://doi.org/10.1111/j.2044-8309.1997.tb01151.x

Steinmetz, J., \& Mussweiler, T. (2011). Breaking the ice: How physical warmth shapes social comparison consequences. Journal of Experimental Social Psychology, 47, 1025-1028. https://doi.org/10.1016/j.jesp.2011.03.022

Todorov, A., Dotsch, R., Porter, J. M., Oosterhof, N. N., \& Falvello, V. B. (2013). Validation of data-driven computational models of social perception of faces. Emotion, 13, 724. https://doi.org/10.1037/a0032335

Todorov, A., Olivola, C. Y., Dotsch, R., \& Mende-Siedlecki, P. (2015). Social attributions from faces: Determinants, consequences, accuracy, and functional significance. Annual Review of Psychology, 66, 519-545. https://doi.org/10.1146/annurev-psych-113011-143831

Venables, B., Hornik, K., \& Maechler, M. (2016). Polynom: A collection of functions to implement a class for univariate polynomial manipulations. R package version 1.3-9. https://CRAN.R-project.org/package=polynom

Wedell, D. H., Parducci, A., \& Geiselman, R. E. (1987). A formal analysis of ratings of physical attractiveness: Successive contrast and simultaneous assimilation. Journal of Experimental Social Psychology, 23, 230-249. https://doi.org/10.1016/00221031(87)90034-5 
Willis, J., \& Todorov, A. (2006) First impressions: Making up your mind after 100 ms exposure to a face. Psychological Science, 17, 592-598. https://doi.org/10.1111/j.1467$9280.2006 .01750 . x$

Wood, S., \& Scheipl, F. (2017). Gamm4: Generalized additive mixed models using 'mgcv' and 'Ime4'. R package version 0.2-5. https://CRAN.R-project.org/package=gamm4

World Medical Association. (2001). World Medical Association Declaration of Helsinki. Ethical principles for medical research involving human subjects. Bulletin of the World Health Organization, 79, 373 - 374. https://www.ncbi.nlm.nih.gov/pmc/articles/PMC2566407/ Zeileis, A. (2004). "Econometric computing with HC and HAC covariance matrix estimators." Journal of Statistical Software, 11(10), 1-17. http://doi.org/10.18637/jss.v011.i10 


\section{Appendix A}

\section{Fortschrittsbalken}

Wie oft setzt die zu beurteilende Person seine Meinung durch im Verlauf von 6 Monaten?
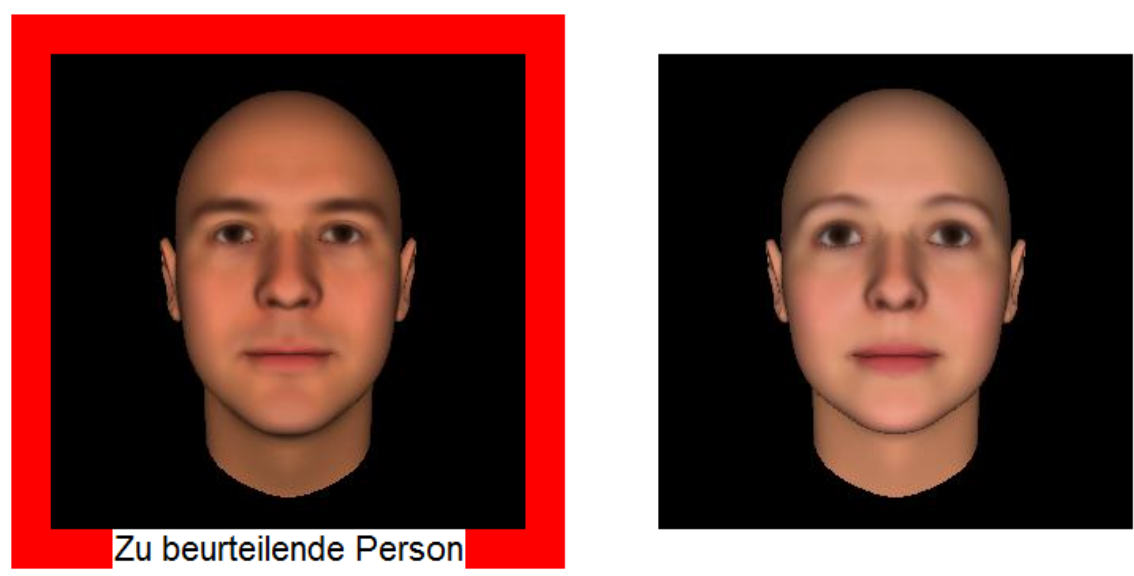

Enter 


\section{Appendix B}
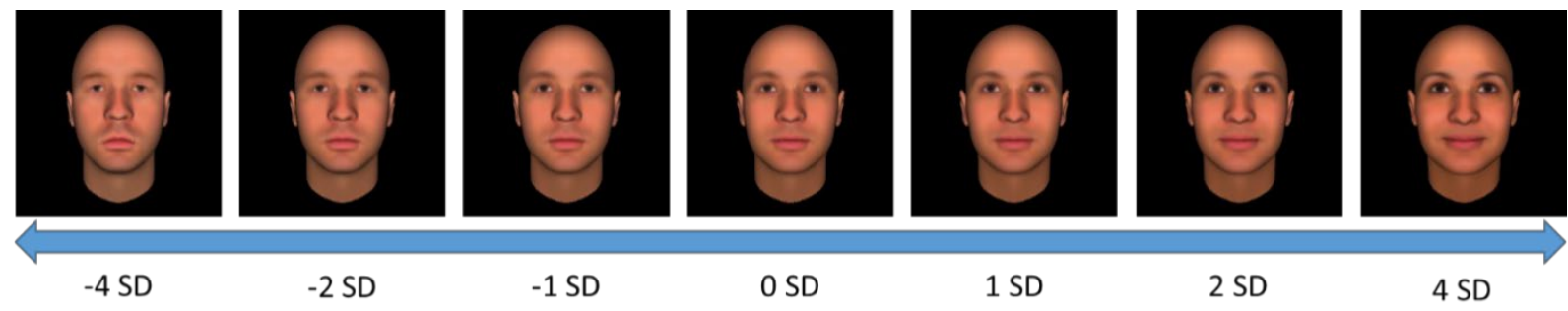

$1 S D$

$2 \mathrm{SD}$

4 SD

Extraversion
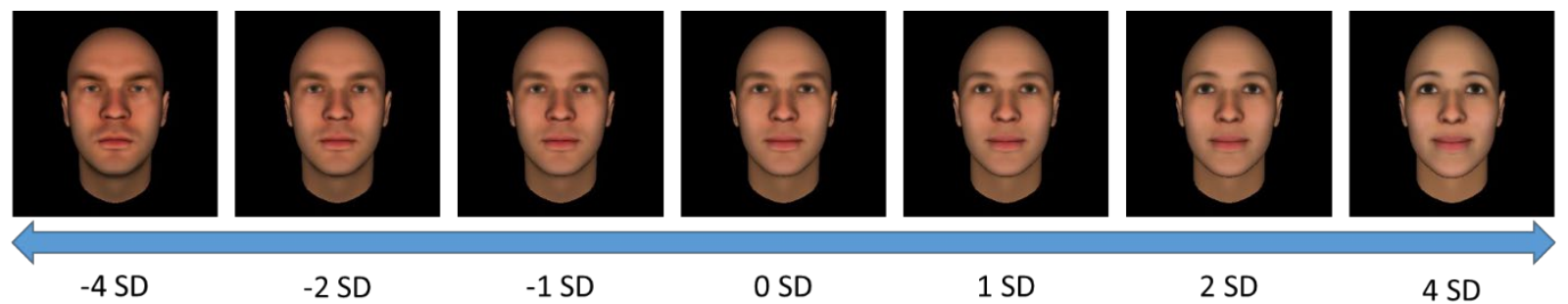

$-1 S D$

O SD

$1 \mathrm{SD}$

$2 \mathrm{SD}$

Trustworthiness
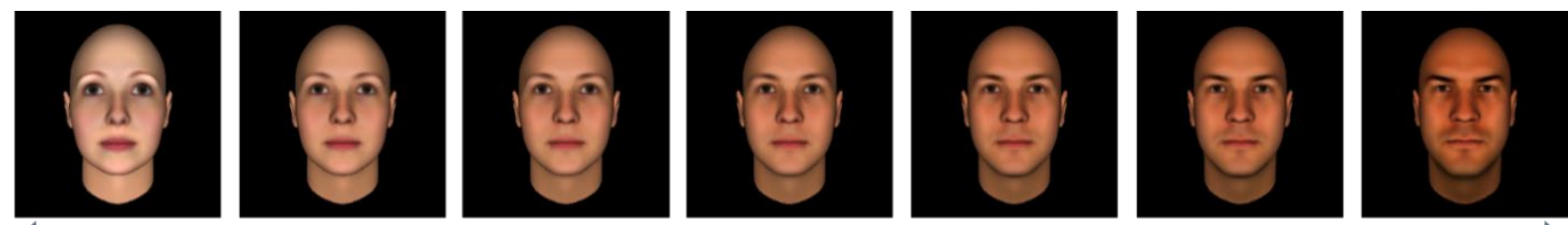

$-4 S D$

$-2 S D$

$-1 S D$

O SD

$1 \mathrm{SD}$

2 SD

4 SD

Dominance 


\section{Appendix C}

Table 1A.

Items selected to represent Dominant and Submissive behaviors for use in Study $2 a$. Dominant:

1. How many times does the target enforce his opinion in 6 month?

2. How many times does the target take the lead in a group in 6 months?

3. How many times does the target try to persuade people to think like they do in 6 months?

Submissive:

1. How many times does the target follow someone else's instructions in 6 months?

2. How many times does the target try to speak quietly in 6 months?

3. How many times does the target try to avoid making decisions in 6 months?

Table 2A.

Items selected to represent Trustworthy and Untrustworthy behaviors for use in Study $2 b$. Trustworthy:

1. How many times does the target keep his promises in 6 months?

2. How many times does the target return things that they have borrowed in 6 months?

3. How many times does the target act friendly and smile at people in 6 months?

Untrustworthy:

1. How many times does the target not show up to an appointment in 6 month?

2. How many times does the target try to manipulate or trick other people in 6 months?

3. How many times does the target do something illegal in 6 months? 